\title{
FLORA Y VEGETACIÓN DE LA SIERRA DE ÓRGANOS, MUNICIPIO DE SOMBRERETE, ZACATECAS, MÉXICO
}

\author{
E. David Enríquez Enríquez \\ Universidad Autónoma de Zacatecas, Facultad de Agronomía \\ km 15.5, carretera Zacatecas-Guadalajara, 98171 Zacatecas, Zacatecas \\ Stephen D. Koch \\ Colegio de Postgraduados, Especialidad de Botánica \\ Instituto de Recursos Naturales, 56230 Montecillo, Edo. de México \\ M. Socorro González-Elizondo
}

CIIDIR-IPN Unidad Durango, Apartado postal 738, 34000 Durango, Durango.

\section{RESUMEN}

La Sierra de Órganos, localizada en la parte occidental del estado de Zacatecas, México, se destaca por sus formaciones columnares de riolita esculpidas por la erosión. Fue decretada como Parque Nacional el 27 de noviembre de 2000. De la colección e identificación de la flora vascular del área resultó una lista florística que incluye 406 especies, 254 géneros y 75 familias; de estas últimas, las más diversas son Asteraceae, Poaceae y Fabaceae. Con base en fotointerpretación, colectas botánicas y 29 muestreos cuantitativos, se reconocen nueve tipos de vegetación definidos por su fisonomía y especies dominantes: bosque de Quercus, bosque de Pinus, bosque de Pinus-Quercus, bosque de Pinus-Juniperus, vegetación de arroyos pedregosos, vegetación de peñascos, matorral de Mimosa-Opuntia, pastizal y vegetación acuática y subacuática. Zacatecas.

Palabras clave: conservación, flora, México, Sierra de Órganos, vegetación,

\section{ABSTRACT}

The Sierra de Organos, located in the western part of the state of Zacatecas, Mexico, is a mountainous area consisting principally of rhyolitic pinnacles. The area was declared a National Park in November 2000. The collection and identification of the vascular flora of the area resulted in a floristic list that includes 406 species, 254 genera, and 75 families. The most diverse families are Asteraceae, Poaceae and Fabaceae. On the basis of physiognomy and dominant species we recognize nine vegetation types, which were delimited and mapped with the aid of aerial photographs, botanical collections, and 29 sample plots. The vegetation types include Quercus woods, Pinus woods, mixed Pinus and 
Quercus woods, mixed Pinus and Juniperus woods, vegetation of rocky streams, vegetation of rock pinnacles, Mimosa and Opuntia scrub, grassland, and aquatic-subaquatic vegetation.

Key words: conservation, floristics, Mexico, Sierra de Organos, vegetation, Zacatecas.

\section{INTRODUCCIÓN}

El acelerado proceso de destrucción de ecosistemas y de extinción de especies que se está dando en nuestros días amenaza a la conservación de la biodiversidad y dificulta el manejo sustentable de los recursos. Una de las medidas para fundamentar programas de conservación es evaluar la diversidad biológica, tanto en el ámbito específico como de ecosistemas, a través de un inventario de la flora nacional que incluya datos referentes a la composición de los tipos de vegetación y a la abundancia de los individuos (Riba, 1995).

Zacatecas es uno de los estados con más bajo índice de colección botánica y con mayor rezago en cuanto al conocimiento de su flora (Dávila y Sosa,1994), por lo que es conveniente intensificar los estudios tendientes a conocer su diversidad vegetal. Entre los trabajos florísticos modernos que se han realizado en el estado, o que incluyen partes de su territorio, destacan los llevados a cabo por McVaugh (1983, 1984, 1985, 1987, 1989, 1992, 1993) en la región de los Cañones, en el suroeste del estado, como parte de la flora de Nueva Galicia. Para esa misma región, Enríquez (1998) censó la flora del cerro La Cantarilla. Nieves et al. (1999) elaboraron un inventario de las plantas vasculares del norte de Jalisco y zonas adyacentes de Durango, Nayarit y Zacatecas; Balleza (1992) estudió las gramíneas en el estado y, Balleza y Villaseñor (2002) presentaron una relación de las Asteraceae de Zacatecas y documentaron sus patrones de distribución. Estudios de vegetación fueron realizados por Rzedowski (1957), Guzmán y Vela (1960), Rzedowski y McVaugh (1966), Anónimo (1980a), Anónimo (1981) y González (1998). Trabajos de carácter ecológico han sido presentados por Claverán (1961), González E. (1975), Aldrete (1981) y Luna et al. (1997). La contribución más relacionada con la Sierra de Órganos, por su relativa cercanía geográfica, es la referente a la vegetación de la Reserva de la Biosfera "La Michilía", en Durango, de González-Elizondo et al. (1993). Ninguno de los trabajos señalados incluye a la mencionada Sierra de Órganos.

El presente artículo tiene como objetivos determinar la diversidad florística en la Sierra de Órganos, registrar cómo se distribuye ésta en sus comunidades vegetales y describir los principales tipos de vegetación, destacando su estructura y composición.

La Sierra de Órganos es de interés ecológico y biogeográfico debido a su posición limítrofe entre las provincias florísticas de la Altiplanicie y la Sierra Madre Occidental (Rzedowski, 1978; Anónimo, 1980d). Dado que en México las zonas áridas compiten por el segundo lugar en lo que se refiere a riqueza florística total 
después de los bosques de pino y encino (Rzedowski, 1993), se espera que la flora de la zona estudiada presente una diversidad relativamente alta. La Sierra de Órganos consiste en parte de macizos rocosos en forma de columnas producidos por la erosión, lo que le confiere gran belleza escénica e interés turístico, y fue declarada Parque Nacional por el Gobierno Federal el 27 de noviembre de 2000, en una superficie de 1124 ha.

\section{DESCRIPCIÓN DEL ÁREA}

El área de estudio, dentro de la cual se encuentra el Parque Nacional Sierra de Órganos, comprende una superficie de 4792.6 ha y se ubica en el occidente del Estado de Zacatecas en el municipio de Sombrerete, $20 \mathrm{~km}$ al noroeste de la ciudad del mismo nombre. Forma parte de la Sierra de Santa Lucía, en un ramal de la Sierra Madre Occidental en los límites con la Altiplanicie Mexicana, entre $23^{\circ} 44^{\prime} 58^{\prime \prime}$ y $23^{\circ} 48^{\prime} 29^{\prime \prime}$ de latitud norte y $103^{\circ} 45^{\prime} 51^{\prime \prime}$ y $103^{\circ} 49^{\prime} 36^{\prime \prime}$ de longitud oeste. Sus extremos en elevación sobre el nivel del mar son 2170 y $2560 \mathrm{~m}$, y se localizan en el arroyo La Ciénega y el Cerro Alto, respectivamente. Los valles intermontanos tienen una elevación promedio de $2300 \mathrm{~m}$ (Fig. 1). El clima predominante en la región es seco BS $\mathrm{kw}$, sin condición de canícula y en las partes altas el clima es templado $\mathrm{C}\left(\mathrm{w}_{0}\right)$ (Anónimo, 1980b). La caseta meteorológica más

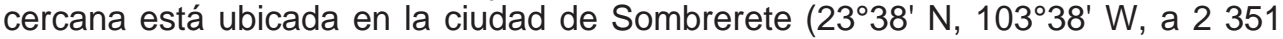
m s.n.m.). Con datos de esa estación Ortiz (1990) calculó la temperatura media anual $\left(16.6^{\circ} \mathrm{C}\right)$ y la precipitación media anual $(613 \mathrm{~mm})$, valores que se consideran próximos a los correspondientes a la Sierra de Órganos.

La Sierra Madre Occidental surgió como resultado de la subducción de la placa Paleo Pacífica (hipotética) bajo la placa Norteamericana, lo que originó una serie de serranías volcánicas (Ferrusquía-Villafranca, 1993). Una de estas serranías es la Sierra Santa Lucía. Las riolitas de color café rojizo de esta Sierra tienen una edad probable del Cenozoico medio (Shannon y Kramer, 1973); Anónimo (1980c) señala que las rocas ígneas extrusivas son del Terciario, las sedimentarias del Cretácico y los suelos del Cuaternario. La mayor parte de las rocas del área de estudio son ígneas extrusivas ácidas con predominancia de riolita, formada de feldespatos y cuarzo abundante. En la porción suroeste existen rocas sedimentarias de tipo conglomerado y caliza (Anónimo, 1979).

El área de estudio está dividida por el arroyo La Ciénega (Apéndice 2) en dos regiones definidas por el tipo de substrato y la erosión. En la porción ubicada hacia el suroeste del arroyo se encuentra el área menos erosionada, con superficies onduladas donde predominan las rocas calizas. La parte ubicada al norte y noreste del arroyo se compone de conjuntos de cerros de riolita, algunos muy erosionados y formados de columnas y de grandes macizos rocosos que corren principalmente de norte a sur. Entre estos cerros existen valles intermontanos con pendientes suaves; los valles están cortados por arroyos de lechos arenosos en su parte baja y pedregosos en la alta. Ningún arroyo tiene corrientes permanentes de agua. 


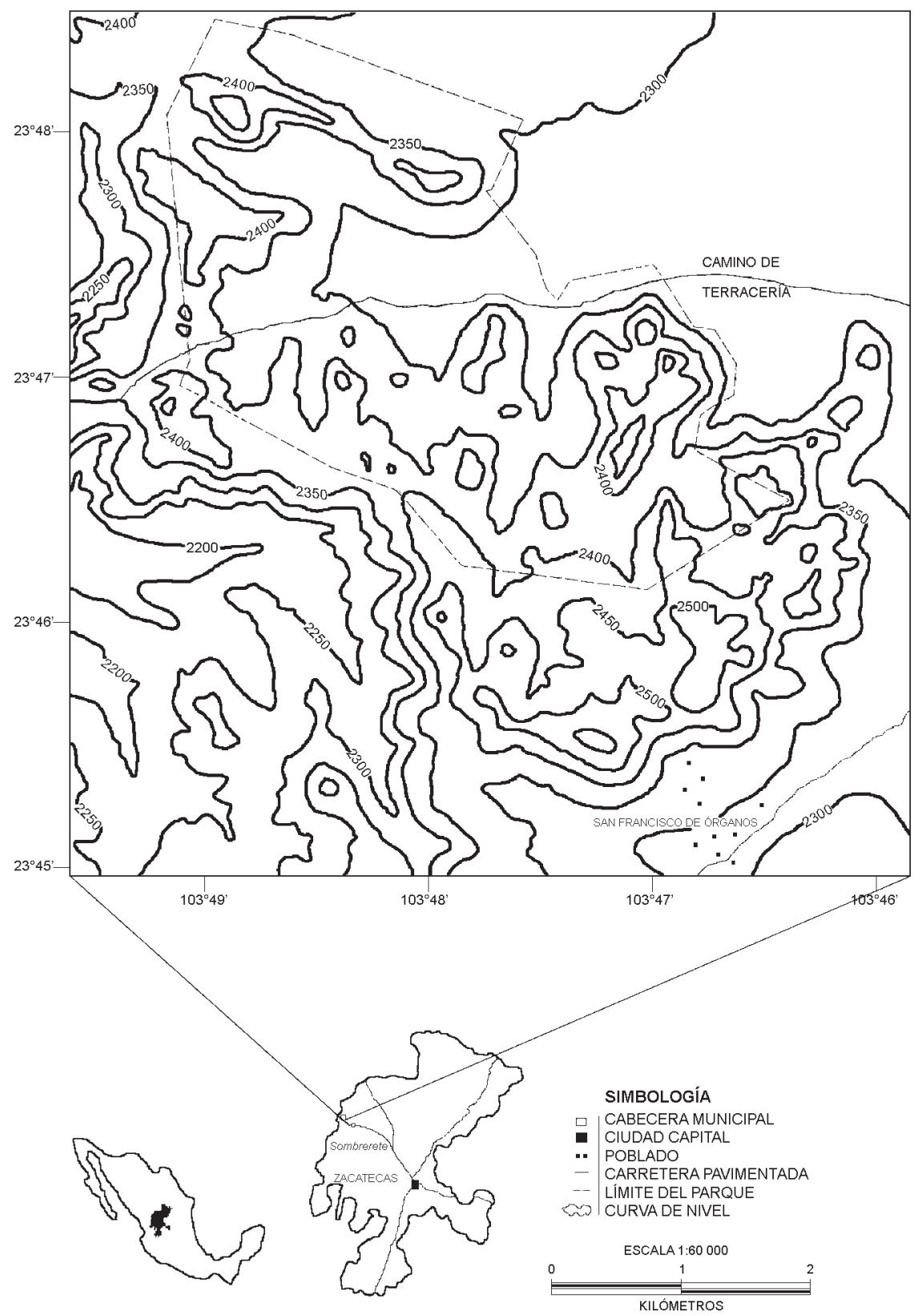

Figura 1. Localización y mapa hipsométrico de la Sierra de Órganos, municipio de Sombrerete, Zacatecas, México. 
Los suelos son litosoles eútricos con textura media en los lomeríos y terrenos montuosos con pendientes de 8 a $20 \%$ y xerosoles háplicos de textura media en terrenos planos a ligeramente ondulados con pendientes menores de 8\% (Anónimo, 1971).

\section{MÉTODOS}

Riqueza de especies

Para conocer la riqueza de especies se exploró el área de estudio y se recolectaron ejemplares de herbario durante las estaciones del año. Los muestreos de vegetación y flora se realizaron de agosto de 1999 a septiembre de 2001. Los especímenes se identificaron con ayuda de claves y descripciones de floras regionales, así como de revisiones y monografías de grupos taxonómicos. De manera complementaria se acudió a especialistas en algunas familias para la determinación de los materiales correspondientes. Los ejemplares identificados se cotejaron con los depositados en los herbarios del Centro Interdisciplinario de Investigación para el Desarrollo Integral Regional (CIIDIR) del Instituto Politécnico Nacional, Unidad Durango; del Colegio de Postgraduados (CHAPA) y del Instituto de Biología (MEXU) de la Universidad Nacional Autónoma de México. El primer juego de la colección quedó depositado en CHAPA y el segundo en el herbario de la Universidad Autónoma de Zacatecas (HUAZ).

Vegetación

Para el reconocimiento de los tipos de vegetación se aplicó un criterio fisonómico-florístico. En cada una de las comunidades vegetales se establecieron áreas de muestreo con objeto de determinar el valor de importancia de las especies. Con el propósito de tomar datos de cobertura y densidad de árboles y arbustos se establecieron 29 parcelas de $2 \times 100 \mathrm{~m}\left(200 \mathrm{~m}^{2}\right)$, con lo que la superficie total muestreada suma $5800 \mathrm{~m}^{2}$. A fin de ubicar los sitios de muestreo se marcó la carta topográfica del área de estudio con una cuadrícula con separación de 0.5 minutos, estableciendo por lo menos un sitio de muestreo dentro de cada rectángulo de 0.5 minutos de lado; se consideró además la topografía, incluyendo las áreas de peñascos, arroyos, valles intermontanos, cañadas y laderas.

El número de individuos por unidad de área corresponde a la densidad. La frecuencia se obtuvo a partir del número de parcelas en que apareció la especie en cuestión. La cobertura se consideró como el área ocupada por cada especie. Para árboles y arbustos fue calculada a partir de la medición de dos diámetros perpendiculares de las copas utilizando la siguiente fórmula:

$$
\left.\mathrm{C}=\left[\left(\mathrm{d}_{1}+\mathrm{d}_{2}\right) /\right]_{4}\right]^{2} \pi
$$


donde: $\quad \mathrm{C}=$ Cobertura

$\mathrm{d}_{1}=$ Primer diámetro de cobertura de la copa

$d_{2}=$ Segundo diámetro de cobertura de la copa que cruza en forma perpendicular a $d_{1}$

Se calculó el valor de importancia (V.I.) de cada especie de la siguiente manera: V.I.= densidad relativa (número de individuos por especie / total de individuos de las especies x 100) + frecuencia relativa (número de veces que se encontró una especie en el muestreo / total de veces que se econtraron las especies en el muestreo $\times 100)$ + cobertura relativa (área de cada especie / área total de especies x 100) (Curtis y McIntosh, 1951).

Los datos de cobertura y densidad de plantas herbáceas se obtuvieron mediante el establecimiento de 10 subparcelas de $1 \mathrm{~m}^{2}$ ubicadas dentro de cada parcela de muestreo de $2 \times 100 \mathrm{~m}$. Las subparcelas se colocaron sobre una de las líneas de $100 \mathrm{~m}$, separadas por una distancia de $10 \mathrm{~m}$. Para calcular la densidad se hizo el conteo de todos los individuos de cada especie en cada subparcela y se obtuvo un promedio de las 10 subparcelas. Con los datos de densidad se evaluó la cobertura. Para ello se estimó visualmente la superficie total cubierta por las plantas herbáceas en cada subparcela; la cobertura por especie se obtuvo mediante el conteo de todos sus individuos, asumiendo que cada uno de ellos tenía el mismo diámetro de follaje, finalmente se obtuvo el promedio de cobertura de cada especie herbácea en las 10 subparcelas.

Adicionalmente, se tomaron datos de la altura media de los estratos arbóreo y arbustivo, así como el diámetro de los troncos a la altura del pecho.

Los valores de importancia fueron agrupados en cuatro categorías mediante cuartiles, tal como se muestra a continuación.

\begin{tabular}{lrrlc}
\hline \multicolumn{2}{c}{ Valor de importancia } & \multicolumn{1}{c}{ Categoría } & $\begin{array}{c}\text { Valor ordinal } \\
\text { asignado }\end{array}$ \\
\hline 0.392 & hasta & 1.147 & Valor de Importancia muy bajo & 1 \\
1.148 & hasta & 2.036 & Valor de Importancia bajo & 2 \\
2.037 & hasta & 4.055 & Valor de Importancia alto & 3 \\
4.056 & hasta & 65.112 & Valor de Importancia muy alto & 4 \\
\hline
\end{tabular}

En el Apéndice 1 se utilizan los valores ordinales asignados para definir el valor de importancia de cada especie. Las especies que no se encontraron dentro de las parcelas, pero sí se observaron en el área, fueron registradas con un valor acumulado de 0 y no se incluyeron en los cálculos de importancia pero sí en los de similitud.

Para la clasificación de la vegetación se utilizó como base la nomenclatura propuesta por Rzedowski (1978) con algunas modificaciones para incluir subcategorías 
encontradas en el parque. Un mapa topográfico a escala 1:50 000 del Instituto Nacional de Geografía y Estadística (INEGI) fue marcado con colores distintivos para cada tipo de vegetación. Se analizaron con un estereoscopio de espejos pares de fotografías aéreas escala 1:25 000 de INEGI a fin de establecer los límites de las unidades de vegetación reconocidas. Las fotografías aéreas fueron tomadas en el año 1969, por lo que se llevaron a cabo recorridos de cotejo para verificar la distribución actual de las unidades de vegetación.

Cálculo de similitud

Con objeto de conocer la similitud florística entre comunidades se usó el índice de Jaccard (Magurran, 1988), que es igual a 100 cuando los sitios comparados comparten las mismas especies, y toma el valor de 0 si no presentan ninguna en común. El índice de Jaccard se calculó con la siguiente fórmula:

$$
C_{j}=[j /(a+b-j)] \times 100
$$

donde: $\quad C_{j}=$ Índice de Jaccard

$\mathrm{j}$ = Número de especies comunes entre dos comunidades

$\mathrm{a}=$ Número de especies de la comunidad a

$\mathrm{b}=$ Número de especies de la comunidad $\mathrm{b}$

Adicionalmente, y con el fin de obtener una representación visual de las relaciones florísticas entre las comunidades estudiadas, se llevó a cabo un análisis a través del programa NTSYS-pc con el método UPGMA (Rohlf, 1998), también aplicando el índice de similitud de Jaccard con el que se obtuvo la matriz de datos.

\section{RESULTADOS}

Riqueza florística

Se identificaron 406 especies pertenecientes a 254 géneros y 75 familias. De éstas, las más diversas son: Asteraceae (102 especies), Poaceae (44), Fabaceae (22), Cyperaceae (15), Euphorbiaceae (11), Lamiaceae (9), Fagaceae (9), Convolvulaceae (10), Rubiaceae (9), Cactaceae (9), Caryophyllaceae (7), Mimosaceae (7) y Adiantaceae (6). En el Apéndice 1 se enumeran las especies encontradas para cada tipo de vegetación, con el valor de importancia correspondiente.

Vegetación

Se reconocen nueve unidades de vegetación para la Sierra de Órganos: bosque de Quercus, bosque de Pinus, bosque de Pinus-Quercus, bosque de Pinus- 
Juniperus, vegetación de arroyos pedregosos, vegetación de peñascos, matorral de Mimosa-Opuntia, pastizal y vegetación acuática y subacuática. La ubicación de las unidades de vegetación se esquematiza en un mapa (Apéndice 2). La vegetación de arroyos pedregosos y la vegetación acuática y subacuática ocupan extensiones muy pequeñas y no se cartografiaron debido a la escala usada.

Para cada una de las unidades de vegetación se presenta una descripción con énfasis en sus condiciones ecológicas y características estructurales y se registran las especies con mayor valor de importancia en cada estrato, anotando primero aquellas que tienen valores más altos. En los Cuadros 1 a 8 se detallan los valores relativos de densidad, cobertura, frecuencia y valor de importancia por estrato para cada comunidad, mostrando parcialmente la lista del estrato herbáceo por razones de espacio.

En algunos casos la forma biológica no está totalmente definida. Así, Loeselia scariosa puede comportarse como herbácea o como subarbustiva, Ageratina rubricaulis como herbácea o arbustiva y Helianthemum glomeratum presenta características intermedias entre ambas formas. Estas especies se registran como parte del estrato herbáceo. Otras más presentan diferente forma biológica dependiendo del lugar donde se desarrollan, por ejemplo, Juniperus deppeana se encuentra como arbustiva en la mayor parte del área de estudio, pero como árbol en la zona de roca caliza (bosque de Pinus-Juniperus).

Los árboles de los bosques del área de estudio presentan en general una densidad baja, con troncos que oscilan entre 20 y $70 \mathrm{~cm}$ de diámetro a la altura del pecho y de manera excepcional hasta $1.2 \mathrm{~m}$. La cubierta herbácea es usualmente densa. No es frecuente la presencia de lianas y las epifitas son escasas.

Bosque de Quercus. Prospera en la zona de rocas riolíticas y conglomerados en las partes más expuestas a la desecación, así como en laderas con suelos someros y pedregosos ubicadas al este del área de estudio y en las mesas de la Peña Larga y de otros cerros situados en la parte noroeste. Cubre una superficie de 84.38 ha dentro del parque. Es una comunidad compuesta de árboles bajos (entre 3 y $4 \mathrm{~m}$ ) y espaciados, de hojas pequeñas y rígidas, asociados con plantas herbáceas que presentan una alta densidad. Las especies arbóreas principales son Quercus grisea y Q. eduardi, con Yucca decipiens y Pinus cembroides ocasionalmente presentes. El estrato arbustivo tiene una altura aproximada de $1 \mathrm{~m}$, y en él predominan Mimosa aculeaticarpa, M. dysocarpa, Arctostaphylos pungens, Juniperus deppeana, Dasylirion wheeleri, Perymenium mendezii, Citharexylum aff. rosei, Opuntia durangensis y $O$. robusta. En el estrato herbáceo destacan Tagetes micrantha, Heterosperma pinnatum, Tridax balbisioides, Salvia axillaris y Sida linearis (Cuadro 1).

Bosque de Pinus. Se desarrolla principalmente sobre rocas riolíticas en las laderas y en algunas superficies planas de la parte norte y oeste del área de estudio, tanto en suelos erosionados como en los profundos. Cubre 47.92 ha dentro del parque. El estrato arbóreo, con alturas de entre 6 y $8 \mathrm{~m}$, está dominado 
Cuadro 1. Índices relativos de densidad, cobertura, frecuencia y valor de importancia de los componentes del bosque de Quercus de la Sierra de Órganos, Sombrerete, Zacatecas.

Densidad
Especie Cobertura Frecuencia Valor de
Relativa
Relativa Relativa Importancia

Estrato arbóreo

Quercus grisea Liebm.

$\begin{array}{rrrr}0.071 & 18.219 & 2.778 & 21.068 \\ 0.016 & 19.764 & 0.926 & 20.706 \\ 0.005 & 0.659 & 0.926 & 1.590 \\ 0.005 & 0.121 & 0.926 & 1.052\end{array}$

Estrato arbustivo y subarbustivo

$\begin{array}{lrrrr}\text { Mimosa aculeaticarpa Ortega } & 0.147 & 9.090 & 2.778 & 12.015 \\ \text { Mimosa dysocarpa Benth. } & 0.038 & 10.567 & 0.926 & 11.531 \\ \text { Arctostaphylos pungens Kunth } & 0.016 & 6.498 & 0.926 & 7.441 \\ \text { Juniperus deppeana Steud. } & 0.016 & 5.049 & 1.852 & 6.917 \\ \text { Dasylirion wheeleri S. Wats. ex Rothr. } & 0.016 & 5.100 & 0.926 & 6.043 \\ \text { Perymenium mendezii DC. } & 0.643 & 3.019 & 1.852 & 5.514 \\ \text { Citharexylum aff. rosei Greenm. } & 0.011 & 0.982 & 1.852 & 2.845 \\ \text { Trixis angustifolia DC. } & 0.027 & 1.655 & 0.926 & 2.608 \\ \text { Opuntia durangensis Britton \& Rose } & 0.027 & 0.305 & 1.852 & 2.184 \\ \text { Opuntia robusta H. Wendl. } & 0.027 & 0.281 & 1.852 & 2.160 \\ \text { Stevia salicifolia Cav. } & 0.218 & 0.073 & 1.852 & 2.143 \\ \text { Telosiphonia hypoleuca (Benth.) Henr. } & 0.909 & 0.089 & 0.926 & 1.923 \\ \text { Ageratina petiolaris (Moc. \& Sessé } & 0.005 & 0.407 & 0.926 & 1.338 \\ \quad \text { ex DC.) R. M. King \& H. Rob. } & & & & \\ \text { Montanoa leucantha (Lag. \& Segura) } & 0.109 & 0.037 & 0.926 & 1.072 \\ \quad \text { S. F. Blake } & & & & \\ \text { Eysenhardtia polystachya (Ortega) Sarg. } & 0.011 & 0.004 & 0.926 & 0.941 \\ \quad & & & & \\ \quad \text { Estrato herbáceo } & & & & \\ & & & & \\ \text { Tagetes micrantha Cav. } & 39.291 & 4.017 & 2.778 & 46.085 \\ \text { Heterosperma pinnatum Cav. } & 8.287 & 2.789 & 1.852 & 12.927 \\ \text { Tridax balbisioides (Kunth) A. Gray } & 6.760 & 2.145 & 2.778 & 11.683 \\ \text { Salvia axillaris Moc. \& Sessé ex Benth. } & 7.996 & 0.780 & 1.852 & 10.628 \\ \text { Sida linearis Cav. } & 5.525 & 1.164 & 2.778 & 9.467 \\ \text { Schkuhria pinnata (Lam.) Kuntze } & 3.707 & 0.987 & 2.778 & 7.472 \\ \text { Aspicarpa hirtella A. Gray } & 3.671 & 0.410 & 1.852 & 5.933 \\ \text { Helianthemum glomeratum Lag. } & 2.435 & 0.342 & 2.778 & 5.555 \\ \text { Selaginella rupincola Underw. } & 3.271 & 1.101 & 0.926 & 5.298 \\ \text { Euphorbia hirta L. } & 0.909 & 0.219 & 2.778 & 3.905 \\ \text { Bouteloua gracilis (Kunth) Lag. } & 1.527 & 0.514 & 1.852 & 3.892 \\ \text { Gomphrena serrata L. } & 1.308 & 0.440 & 1.852 & 3.601 \\ \text { Aristida adscensionis L. } & 0.654 & 0.090 & 2.778 & 3.522 \\ \quad & & & & \end{array}$


Cuadro 1. Continuación.

Especie

Estrato herbáceo

Zornia thymifolia Kunth

Evolvulus alsinoides $\mathrm{L}$.

Desmodium neomexicanum A. Gray

Muhlenbergia rigida (Kunth) Trin.

Setaria geniculata (Lam.) Beauv.

Stevia serrata Cav.

Arenaria lanuginosa (Michx.)

Rohrb.

Allium glandulosum Link \& Otto

Cologania angustifolia Kunth
Densidad

Relativa
Cobertura Frecuencia Valor de

Relativa Relativa Importancia

$\begin{array}{llll}0.981 & 0.330 & 1.852 & 3.163 \\ 1.127 & 0.162 & 1.852 & 3.141 \\ 0.872 & 0.294 & 1.852 & 3.018 \\ 0.872 & 0.294 & 1.852 & 3.018 \\ 0.945 & 0.144 & 1.852 & 2.941 \\ 0.909 & 0.089 & 1.852 & 2.849 \\ 0.545 & 0.183 & 1.852 & 2.581 \\ & & & \\ 0.545 & 0.053 & 1.852 & 2.450 \\ 0.400 & 0.091 & 1.852 & 2.343\end{array}$

esencialmente por Pinus cembroides. En el arbustivo, con alturas de 0.3 a 2 m, se encuentran Calliandra eriophylla, Mimosa aculeaticarpa, Juniperus deppeana y Acacia schaffneri. El estrato herbáceo está dominado por Heterosperma pinnatum, Tagetes micrantha, Schkuhria pinnata, Pectis prostrata, Microchloa kunthii, Eragrostis intermedia y Eleusine multiflora (Cuadro 2).

Cuadro 2. Índices relativos de densidad, cobertura, frecuencia y valor de importancia de los componentes del bosque de Pinus de la Sierra de Órganos, Sombrerete, Zacatecas.

Especie

Estrato arbóreo

Pinus cembroides Zucc.

Quercus eduardi Trel.

Quercus grisea Liebm.

Yucca decipiens Trel.
Densidad Relativa
Cobertura Frecuencia Valor de

Relativa Relativa Importancia

Estrato arbustivo y subarbustivo

Calliandra eriophylla Benth.

Mimosa aculeaticarpa Ortega

Juniperus deppeana Steud.

Acacia schaffneri (S. Watson) F. J. Herm. 0.007

$\begin{array}{lrrr}0.068 & 37.770 & 3.333 & 41.171 \\ 0.001 & 1.111 & 0.556 & 1.668 \\ 0.002 & 0.247 & 1.111 & 1.361 \\ 0.002 & 0.045 & 0.556 & 0.603\end{array}$

0.347

6.518

1.111

7.976

2.263

2.222

4.541

2.014

1.667

3.684

1.676 
Cuadro 2. Continuación.

\begin{tabular}{|c|c|c|c|c|}
\hline Especie & $\begin{array}{l}\text { Densidad } \\
\text { Relativa }\end{array}$ & $\begin{array}{c}\text { Cobertura } \\
\text { Relativa }\end{array}$ & $\begin{array}{c}\text { Frecuencia } \\
\text { Relativa }\end{array}$ & $\begin{array}{l}\text { Valor de } \\
\text { Importancia }\end{array}$ \\
\hline Perymenium mendezii DC. & 0.219 & 0.122 & 1.667 & 2.008 \\
\hline Opuntia durangensis Britton \& Rose & 0.006 & 0.137 & 1.111 & 1.254 \\
\hline $\begin{array}{l}\text { Ageratina pichinchensis (Kunth) } \\
\text { R. M. King \& H. Rob. }\end{array}$ & 0.462 & 0.123 & 0.556 & 1.141 \\
\hline Dasylirion wheeleri S. Wats. ex Rothr. & 0.005 & 0.569 & 0.556 & 1.129 \\
\hline Croton dioicus Cav. & 0.219 & 0.131 & 0.556 & 0.906 \\
\hline Buddleja scordioides Kunth & 0.195 & 0.066 & 0.556 & 0.816 \\
\hline Opuntia robusta $\mathrm{H}$. Wendl. & 0.001 & 0.196 & 0.556 & 0.753 \\
\hline Salvia lycioides A. Gray & 0.073 & 0.044 & 0.556 & 0.672 \\
\hline $\begin{array}{l}\text { Montanoa leucantha (Lag. \& Segura) } \\
\text { S. F. Blake }\end{array}$ & 0.024 & 0.015 & 0.556 & 0.595 \\
\hline Arctostaphylos pungens Kunth & 0.001 & 0.034 & 0.556 & 0.590 \\
\hline Agave parryi Engelm. & 0.006 & 0.022 & 0.556 & 0.584 \\
\hline Eysenhardtia polystachya (Ortega) Sarg. & 0.013 & 0.004 & 0.556 & 0.573 \\
\hline \multicolumn{5}{|l|}{ Estrato herbáceo } \\
\hline Heterosperma pinnatum Cav. & 25.072 & 10.019 & 3.333 & 38.424 \\
\hline Tagetes micrantha Cav. & 12.755 & 7.120 & 2.778 & 22.653 \\
\hline Schkuhria pinnata (Lam.) Kuntze & 10.467 & 5.344 & 3.333 & 19.145 \\
\hline Pectis prostrata Cav. & 7.887 & 4.881 & 1.111 & 13.879 \\
\hline Microchloa kunthii Desv. & 6.913 & 3.811 & 1.667 & 12.391 \\
\hline Eragrostis intermedia Hitchc. & 6.913 & 3.128 & 1.667 & 11.707 \\
\hline Eleusine multiflora Hochst. ex A. Rich. & 7.059 & 2.381 & 0.556 & 9.995 \\
\hline Euphorbia hirta L. & 3.189 & 1.435 & 3.333 & 7.957 \\
\hline Tridax balbisioides (Kunth) A. Gray & 1.704 & 0.996 & 1.667 & 4.367 \\
\hline Dichondra argentea Humb. \& Bonpl. ex Willd. & 0.438 & 0.266 & 3.333 & 4.038 \\
\hline Helianthemum glomeratum Lag. & 0.828 & 0.340 & 2.222 & 3.390 \\
\hline Cyperus seslerioides Kunth & 1.266 & 0.442 & 1.667 & 3.375 \\
\hline Aristida adscensionis L. & 0.365 & 0.204 & 2.222 & 2.792 \\
\hline Salvia axillaris Moc. \& Sessé ex Benth. & 0.536 & 0.321 & 1.667 & 2.524 \\
\hline Muhlenbergia rigida (Kunth) Trin. & 0.536 & 0.279 & 1.667 & 2.481 \\
\hline Desmodium neomexicanum A. Gray & 0.584 & 0.219 & 1.667 & 2.470 \\
\hline Lycurus phleoides Kunth & 0.365 & 0.236 & 1.667 & 2.268 \\
\hline Bouteloua gracilis (Kunth) Lag. & 0.852 & 0.254 & 1.111 & 2.217 \\
\hline Chenopodium graveolens Willd. & 0.365 & 0.152 & 1.667 & 2.184 \\
\hline Plantago linearis Kunth & 0.316 & 0.175 & 1.667 & 2.158 \\
\hline Gomphrena serrata L. & 0.755 & 0.289 & 1.111 & 2.155 \\
\hline
\end{tabular}

Bosque de Pinus-Quercus. Es el tipo de vegetación que ocupa la mayor superficie del parque (551.04 ha). En el área de estudio se desarrolla sobre rocas 
riolíticas en las laderas y en los valles entre los cerros rocosos. El estrato arbóreo, de entre 6 y 7 m, lo forman Pinus cembroides, Quercus grisea y Q. eduardi; en una cañada se presenta también $P$. chihuahuana. En el arbustivo, con altura aproximada de $2 \mathrm{~m}$, se inventariaron Quercus potosina, Arctostaphylos pungens, Mimosa aculeaticarpa, Juniperus deppeana, Garrya wrightii, Perymenium mendezii, Calliandra eriophylla, Opuntia robusta, O. durangensis y Agave parryi. En el estrato herbáceo son abundantes Heterosperma pinnatum, Schkuhria pinnata, Cosmos parviflorus, Tagetes lunulata, T. micrantha y Crusea diversifolia (Cuadro 3).

Cuadro 3. Índices relativos de densidad, cobertura, frecuencia y valor de importancia de los componentes del bosque de Pinus-Quercus de la Sierra de Órganos, Sombrerete, Zacatecas.

Especie

Estrato arbóreo

Pinus cembroides Zucc.

Quercus grisea Liebm.

Quercus eduardi Trel.

Pinus chihuahuana Engelm.

Yucca decipiens Trel.

Estrato arbustivo y subarbustivo

Quercus potosina Trel.

Arctostaphylos pungens Kunth

Mimosa aculeaticarpa Ortega

Juniperus deppeana Steud.

Citharexylum aff. rosei Greenm.

Garrya wrightii Torr.

Perymenium mendezii DC.

Calliandra eriophylla Benth.

Stevia salicifolia Cav.

Opuntia robusta $\mathrm{H}$. Wendl.

Opuntia durangensis Britton \& Rose

Agave parryi Engelm.

Eysenhardtia polystachya (Ortega) Sarg.

Mimosa dysocarpa Benth.

Montanoa leucantha (Lag. \& Segura)

S. F. Blake

Gymnosperma glutinosum (Spreng.) Less.

Salvia lycioides A. Gray
Densidad

Relativa

Cobertura

Frecuencia Valor de

Relativa Relativa Importancia

$\begin{array}{lrrr}2.001 & 27.992 & 2.622 & 32.615 \\ 0.164 & 14.006 & 1.873 & 16.043 \\ 0.906 & 5.617 & 1.124 & 7.647 \\ 0.151 & 1.763 & 0.375 & 2.288 \\ 0.001 & 0.044 & 0.375 & 0.419\end{array}$

$\begin{array}{llll}0.039 & 5.575 & 0.749 & 6.363 \\ 0.008 & 3.262 & 1.124 & 4.394 \\ 0.328 & 1.772 & 2.247 & 4.348 \\ 0.153 & 2.075 & 1.498 & 3.726 \\ 0.005 & 2.050 & 0.749 & 2.804 \\ 0.437 & 0.157 & 1.873 & 2.466 \\ 0.137 & 0.062 & 1.873 & 2.072 \\ 0.070 & 0.921 & 0.749 & 1.740 \\ 0.196 & 0.112 & 1.124 & 1.431 \\ 0.004 & 0.119 & 1.124 & 1.246 \\ 0.005 & 0.418 & 0.749 & 1.172 \\ 0.181 & 0.058 & 0.375 & 0.613 \\ 0.003 & 0.118 & 0.375 & 0.495 \\ 0.002 & 0.086 & 0.375 & 0.463 \\ 0.015 & 0.010 & 0.375 & 0.400 \\ & & & \\ 0.015 & 0.005 & 0.375 & 0.394 \\ 0.015 & 0.002 & 0.375 & 0.392\end{array}$


Cuadro 3. Continuación.

\begin{tabular}{lrrrr} 
Especie & $\begin{array}{c}\text { Densidad } \\
\text { Relativa }\end{array}$ & $\begin{array}{c}\text { Cobertura } \\
\text { Relativa }\end{array}$ & $\begin{array}{c}\text { Frecuencia } \\
\text { Relativa }\end{array}$ & $\begin{array}{c}\text { Valor de } \\
\text { Importancia }\end{array}$ \\
\multicolumn{1}{c}{ Estrato herbáceo } & & & & \\
Heterosperma pinnatum Cav. & & & & \\
Schkuhria pinnata (Lam.) Kuntze & 19.580 & 6.853 & 1.873 & 34.106 \\
Cosmos parviflorus (Jacq.) Pers. & 4.922 & 6.420 & 2.622 & 28.569 \\
Tagetes lunulata Ortega & 4.476 & 2.044 & 0.749 & 8.451 \\
Tagetes micrantha Cav. & 3.685 & 2.220 & 1.498 & 8.018 \\
Crusea diversifolia (Kunth) Anderson & 3.643 & 1.462 & 1.498 & 7.778 \\
Aristida adscensionis L. & 1.880 & 0.613 & 2.247 & 4.603 \\
Helianthemum glomeratum Lag. & 1.216 & 0.468 & 2.622 & 4.306 \\
Euphorbia hirta L. & 1.106 & 0.444 & 2.622 & 4.171 \\
Eleusine multiflora Hochst. ex A. Rich. & 2.785 & 0.891 & 0.375 & 4.050 \\
Tridax balbisioides (Kunth) A. Gray & 1.313 & 0.471 & 2.247 & 4.031 \\
Bouteloua gracilis (Kunth) Lag. & 1.159 & 0.530 & 2.247 & 3.936 \\
Stevia micrantha Lag. & 2.198 & 1.227 & 0.375 & 3.800 \\
Bidens odorata Cav. & 1.835 & 0.349 & 1.498 & 3.682 \\
Nemastylis tenuis (Herb.) S. Watson & 2.439 & 0.780 & 0.375 & 3.593 \\
Cyperus sesleroides Kunth & 0.753 & 0.290 & 2.247 & 3.290 \\
Sisyrinchium tenuifolium & 0.907 & 0.358 & 1.873 & 3.137 \\
$\quad$ Humb. \& Bonpl. ex Willd. & & & & \\
Sida linearis Cav. & 0.602 & 0.285 & 1.873 & 2.759 \\
Peperomia campylotropa Hill & 2.062 & 0.305 & 0.375 & 2.741 \\
& & & & \\
& & & &
\end{tabular}

Bosque de Pinus-Juniperus. Este bosque se encuentra principalmente sobre roca sedimentaria caliza. Se localiza al suroeste del área de estudio, limitando su distribución con el arroyo La Ciénega al norte-noroeste, y es la única de las comunidades estudiadas que no está representada dentro de los límites del parque. Destacan como dominantes Pinus cembroides, Juniperus deppeana y Quercus laeta que forman el estrato superior, con una altura de 4 a $6 \mathrm{~m}$. El arbustivo, con talla de (0.4-)1 a 2 m, se compone de Acacia schaffneri, Forestiera durangensis, Rhus aromatica var. trilobata, Mimosa dysocarpa, Ageratina calaminthifolia, Rhus virens, Cowania mexicana, Opuntia durangensis y Eysenhardtia polystachya. En el estrato herbáceo predominan Sanvitalia procumbens, Euphorbia hirta, Heterosperma pinnatum, Dyssodia papposa, Hilaria cenchroides y Stevia micrantha (Cuadro 4).

Vegetación de arroyos pedregosos. Los arroyos de las partes centrales y altas del área de estudio se caracterizan por sus lechos con afloramiento de roca madre y rocas sueltas. Por encontrarse entre los cerros, por su profundidad y por la existencia de manantiales que perduran la mayor parte del año, la desecación es 
Cuadro 4. Índices relativos de densidad, cobertura, frecuencia y valor de importancia de los componentes del bosque de Pinus-Juniperus de la Sierra de Órganos, Sombrerete, Zacatecas.

Especie

Estrato arbóreo

Pinus cembroides Zucc.

Juniperus deppeana Steud.

Quercus laeta Liebm.

Yucca decipiens Trel.
Densidad

Relativa
Cobertura Frecuencia Valor de

Relativa Relativa Importancia

Estrato arbustivo y subarbustivo

\begin{tabular}{|c|c|c|c|c|}
\hline $\begin{array}{l}\text { Acacia schaffneri (S. Watson) } \\
\text { F. J. Herm. }\end{array}$ & 0.031 & 5.353 & 2.410 & 7.794 \\
\hline Forestiera durangensis Standl. & 0.021 & 3.835 & 2.410 & 6.265 \\
\hline $\begin{array}{l}\text { Rhus aromatica var. trilobata (Nutt.) } \\
\text { A. Gray ex S. Wats. }\end{array}$ & 0.133 & 2.342 & 2.410 & 4.885 \\
\hline Mimosa dysocarpa Benth. & 0.017 & 1.316 & 2.410 & 3.743 \\
\hline $\begin{array}{l}\text { Ageratina calaminthifolia (Kunth) } \\
\text { R. M. King \& H. Rob. }\end{array}$ & 0.550 & 0.249 & 2.410 & 3.209 \\
\hline Rhus virens A. Gray (dos formas) & 0.024 & 0.573 & 2.410 & 3.007 \\
\hline Cowania mexicana D. Don & 0.017 & 0.550 & 2.410 & 2.977 \\
\hline Opuntia durangensis Britton \& Rose & 0.007 & 0.180 & 2.410 & 2.597 \\
\hline Salvia greggii Gray & 0.075 & 0.036 & 2.410 & 2.520 \\
\hline Eysenhardtia polystachya (Ortega) Sarg. & 0.024 & 0.462 & 1.205 & 1.691 \\
\hline Gymnosperma glutinosum (Spreng.) Less. & 0.205 & 0.106 & 1.205 & 1.516 \\
\hline Opuntia robusta $\mathrm{H}$. Wendl. & 0.003 & 0.119 & 1.205 & 1.327 \\
\hline Baccharis pteronioides DC. & 0.068 & 0.035 & 1.205 & 1.309 \\
\hline Garrya wrightii Torr. & 0.014 & 0.000 & 1.205 & 1.219 \\
\hline Agave parryi Engelm. & 0.007 & 0.000 & 1.205 & 1.212 \\
\hline Citharexylum aff. rosei Greenm. & 0.003 & 0.000 & 1.205 & 1.208 \\
\hline Amelanchier denticulata (Kunth) Koch & 0.003 & 0.000 & 1.205 & 1.208 \\
\hline
\end{tabular}

\section{Estrato herbáceo}

Sanvitalia procumbens Lam.

Euphorbia hirta L.

Heterosperma pinnatum Cav.

Dyssodia papposa (Vent.) Hitchc.

Hilaria cenchroides Kunth

Stevia micrantha Lag.

Loeselia coerulea (Cav.) G. Don

Bouteloua curtipendula (Michx.) G. S. Torr.

Crusea diversifolia (Kunth) Anderson

$\begin{array}{rrrr}0.103 & 21.510 & 2.410 & 24.022 \\ 0.065 & 12.144 & 2.410 & 14.618 \\ 0.075 & 3.204 & 2.410 & 5.689 \\ 0.007 & 0.000 & 1.205 & 1.212\end{array}$

$\begin{array}{rrrr}22.758 & 11.510 & 2.410 & 36.678 \\ 12.917 & 5.977 & 2.410 & 21.304 \\ 11.482 & 5.439 & 2.410 & 19.330 \\ 7.654 & 3.891 & 2.410 & 13.955 \\ 6.834 & 3.109 & 2.410 & 12.353 \\ 6.698 & 3.477 & 1.205 & 11.380 \\ 4.852 & 2.302 & 2.410 & 9.564 \\ 4.306 & 2.153 & 1.205 & 7.663 \\ 3.212 & 1.523 & 2.410 & 7.145\end{array}$


Cuadro 4. Continuación.

\begin{tabular}{lcccc}
\hline Especie & $\begin{array}{c}\text { Densidad } \\
\text { Relativa }\end{array}$ & $\begin{array}{c}\text { Cobertura } \\
\text { Relativa }\end{array}$ & $\begin{array}{c}\text { Frecuencia } \\
\text { Relativa }\end{array}$ & $\begin{array}{c}\text { Valor de } \\
\text { Importancia }\end{array}$ \\
Desmodium neomexicanum A. Gray & 2.939 & 1.422 & 2.410 & 6.771 \\
Acalypha neomexicana Müll. Arg. & 2.597 & 1.302 & 2.410 & 6.308 \\
Cologania angustifolia Kunth & 1.982 & 0.895 & 2.410 & 5.286 \\
Dalea humilis G. Don & 1.777 & 0.881 & 2.410 & 5.068 \\
Aspicarpa hirtella A. Gray & 1.367 & 0.648 & 2.410 & 4.424 \\
Bothriochloa barbinodis (Lag.) Herter & 1.093 & 0.557 & 2.410 & 4.061 \\
Sanvitalia angustifolia Engelm. ex A. Gray & 0.547 & 0.253 & 2.410 & 3.209 \\
Gomphrena serrata L. & 0.478 & 0.223 & 2.410 & 3.111 \\
Aristida adscensionis L. & 1.162 & 0.515 & 1.205 & 2.882 \\
Tagetes micrantha Cav. & 1.093 & 0.485 & 1.205 & 2.783 \\
& & & & \\
\hline
\end{tabular}

menor que en los arroyos arenosos de las partes bajas, y la estructura y composición de su flora difiere de la de estos últimos. Mientras que la flora de los arroyos arenosos es similar a la de sus áreas adyacentes, la de las corrientes pedregosas es más peculiar. Aunque en el estrato arbóreo se presentan Pinus cembroides, Quercus grisea y Quercus eduardi, los árboles tienen una altura menor que en los bosques adyacentes y muestran una baja densidad y cobertura, al igual que las herbáceas acompañantes. Adicionalmente, ahí se desarrollan elementos como Fraxinus velutina y Prunus serotina que, aunque escasos, son exclusivos de esta comunidad. En el estrato arbustivo se encuentran Quercus potosina, Arctostaphylos pungens, Juniperus flaccida y Perymenium mendezii. Los componentes herbáceos más importantes son Lobelia laxiflora, Tagetes lunulata, Tagetes micrantha, Heterosperma pinnatum, Bidens odorata, Selaginella pallescens, Schkuhria pinnata, Cosmos parviflorus, Cheilanthes kaulfussii, Juncus arcticus, Echeandia flexuosa, Melampodium sericeum, Commelina dianthifolia, Acalypha neomexicana y Cologania angustifolia (Cuadro 5).

Cuadro 5. Índices relativos de densidad, cobertura, frecuencia y valor de importancia de los componentes de la vegetación de arroyos pedregosos de la Sierra de Órganos, Sombrerete, Zacatecas.

Especie

Estrato arbóreo

Pinus cembroides Zucc.

Quercus grisea Liebm. $\begin{array}{ccc}\text { Densidad } & \text { Cobertura Frecuencia Valor de } \\ \text { Relativa } & \text { Relativa Relativa Importancia }\end{array}$ 
Cuadro 5. Continuación.

\begin{tabular}{|c|c|c|c|c|}
\hline Quercus eduardi Trel. & 0.059 & 12.363 & 2.941 & 15.363 \\
\hline Yucca decipiens Trel. & 0.020 & 0.158 & 2.941 & 3.119 \\
\hline \multicolumn{5}{|l|}{ Estrato arbustivo y subarbustivo } \\
\hline Quercus potosina Trel. & 0.196 & 11.644 & 2.941 & 14.781 \\
\hline Arctostaphylos pungens Kunth & 0.127 & 3.058 & 2.941 & 6.126 \\
\hline Juniperus flaccida Schltdl. & 0.020 & 4.509 & 1.471 & 5.999 \\
\hline Baccharis salicifolia (Ruiz \& Pav.) Pers. & 0.029 & 0.299 & 2.941 & 3.269 \\
\hline Perymenium mendezii DC. & 0.784 & 0.594 & 1.471 & 2.848 \\
\hline Agave parryi Engelm. & 0.392 & 0.297 & 1.471 & 2.159 \\
\hline Bouvardia scabrida Mart. \& Gal. & 0.392 & 0.297 & 1.471 & 2.159 \\
\hline Opuntia robusta $\mathrm{H}$. Wendl. & 0.039 & 0.473 & 1.471 & 1.983 \\
\hline Buddleja cordata Kunth & 0.020 & 0.420 & 1.471 & 1.910 \\
\hline Brickellia secundiflora (Lag.) A. Gray & 0.357 & 0.040 & 1.471 & 1.868 \\
\hline $\begin{array}{l}\text { Montanoa leucantha (Lag. \& Segura) } \\
\text { S. F. Blake }\end{array}$ & 0.357 & 0.040 & 1.471 & 1.868 \\
\hline Mimosa aculeaticarpa Ortega & 0.020 & 0.254 & 1.471 & 1.744 \\
\hline \multicolumn{5}{|l|}{ Estrato herbáceo } \\
\hline Tagetes Iunulata Ortega & 21.219 & 4.937 & 4.412 & 30.568 \\
\hline Lobelia laxiflora Kunth & 14.965 & 1.687 & 1.471 & 18.123 \\
\hline Tagetes micrantha Cav. & 9.799 & 1.559 & 1.471 & 12.829 \\
\hline Heterosperma pinnatum Cav. & 5.291 & 2.600 & 2.941 & 10.833 \\
\hline Bidens odorata Cav. & 6.254 & 0.717 & 2.941 & 9.912 \\
\hline $\begin{array}{l}\text { Selaginella pallescens (Presl) } \\
\text { Spring. }\end{array}$ & 4.703 & 3.562 & 1.471 & 9.736 \\
\hline Schkuhria pinnata (Lam.) Kuntze & 4.419 & 0.662 & 2.941 & 8.023 \\
\hline Cosmos parviflorus (Jacq.) Pers. & 4.116 & 0.889 & 2.941 & 7.946 \\
\hline Cheilanthes kaulfussii Kunze & 5.346 & 0.606 & 1.471 & 7.422 \\
\hline Juncus arcticus Willd. & 1.515 & 0.216 & 2.941 & 4.673 \\
\hline Echeandia flexuosa Greenm. & 2.744 & 0.437 & 1.471 & 4.651 \\
\hline Melampodium sericeum Lag. & 0.980 & 0.625 & 2.941 & 4.546 \\
\hline Commelina dianthifolia Delile & 1.141 & 0.165 & 2.941 & 4.247 \\
\hline Acalypha neomexicana Müll. Arg. & 2.352 & 0.374 & 1.471 & 4.197 \\
\hline Cologania angustifolia Kunth & 0.784 & 0.359 & 2.941 & 4.084 \\
\hline Setaria geniculata (Lam.) Beauv. & 0.731 & 0.209 & 2.941 & 3.881 \\
\hline Verbesina pantoptera S. F. Blake & 0.570 & 0.317 & 2.941 & 3.828 \\
\hline Helianthemum glomeratum Lag. & 1.960 & 0.312 & 1.471 & 3.742 \\
\hline Salvia axillaris Moc. \& Sessé ex Benth. & 1.176 & 0.891 & 1.471 & 3.537 \\
\hline Cheilanthes bonariensis (Willd.) Proctor & 0.784 & 0.594 & 1.471 & 2.848 \\
\hline Crusea diversifolia (Kunth) Anderson & 0.784 & 0.594 & 1.471 & 2.848 \\
\hline Erigeron janivultus G. L. Nesom & 0.784 & 0.594 & 1.471 & 2.848 \\
\hline Muhlenbergia speciosa Vasey & 0.784 & 0.594 & 1.471 & 2.848 \\
\hline Pellaea cordifolia (Sessé \& Moc.) A. R. Smith & h 0.713 & 0.081 & 1.471 & 2.265 \\
\hline
\end{tabular}


Vegetación de peñascos. Este tipo de vegetación se encuentra en los cerros más erosionados del área de estudio, sobre rocas riolíticas que forman columnas, montículos y paredes peñascosas con escasos lugares en los que hay acumulación de suelo. En el parque ocupa una extensión de 179.68 ha.

Abundan las especies herbáceas como Selaginella rupincola y Tillandsia fresnilloensis, que cubren la superficie de las rocas; en los sitios donde se acumula el suelo se encuentran otros elementos herbáceos como Schkuhria pinnata, Tagetes micrantha, Cheilanthes bonariensis, Heterosperma pinnatum, Cosmos parviflorus, Tagetes lunulata, Cyperus sesleroides, Arenaria lycopodioides, Plantago linearis, Commelina dianthifolia, Crusea diversifolia, Tridax balbisioides, Polypodium thyssanolepis, Muhlenbergia rigida y Helianthemum glomeratum. También se presentan Agave parryi, A. schidigera y abundantes cactáceas como Echinocereus polyacanthus, Mammillaria gummifera y $M$. moelleriana. Esta área es el hábitat principal de especies como Coreopsis rudis, Eutetras sp. y Tillandsia fresnilloensis. De forma muy dispersa se encuentran Buddleja cordata, Opuntia robusta, Pinus cembroides, Quercus eduardi y Q. potosina (Cuadro 6).

Cuadro 6. Índices relativos de densidad, cobertura, frecuencia y valor de importancia de los componentes de la vegetación de peñascos de la Sierra de Órganos, Sombrerete, Zacatecas.

Especie

Estrato arbóreo

Pinus cembroides Zucc.

Quercus eduardi Trel.

Yucca decipiens Trel.
Densidad

Relativa
Cobertura Frecuencia Valor de

Relativa Relativa Importancia

Estrato arbustivo y subarbustivo

Agave parryi Engelm.

Arctostaphylos pungens Kunth

Quercus potosina Trel.

Montanoa leucantha (Lag. \& Segura)

S. F. Blake

Stevia salicifolia Cav.

Buddleja cordata Kunth

Bouvardia scabrida Mart. \& Gal.

Citharexylum aff. rosei Greenm.

Opuntia robusta $\mathrm{H}$. Wendl.

Mimosa aculeaticarpa Ortega

Opuntia durangensis Britton \& Rose

$\begin{array}{llll}0.017 & 2.211 & 4.000 & 6.229 \\ 0.004 & 1.983 & 2.000 & 3.988 \\ 0.004 & 0.027 & 1.000 & 1.031\end{array}$

$\begin{array}{llll}1.545 & 0.823 & 2.000 & 4.368\end{array}$

$\begin{array}{llll}0.013 & 1.894 & 2.000 & 3.907\end{array}$

$\begin{array}{llll}0.017 & 2.693 & 1.000 & 3.710\end{array}$

$\begin{array}{llll}0.172 & 0.081 & 3.000 & 3.252\end{array}$

$\begin{array}{llll}0.601 & 0.598 & 2.000 & 3.199\end{array}$

$\begin{array}{llll}0.009 & 0.895 & 2.000 & 2.904\end{array}$

$\begin{array}{llll}0.172 & 0.087 & 2.000 & 2.259\end{array}$

$\begin{array}{llll}0.013 & 0.732 & 1.000 & 1.745\end{array}$

$\begin{array}{llll}0.180 & 0.205 & 1.000 & 1.385\end{array}$

$\begin{array}{llll}0.004 & 0.220 & 1.000 & 1.225\end{array}$

$\begin{array}{llll}0.004 & 0.001 & 1.000 & 1.005\end{array}$ 
Cuadro 6. Continuación.

\begin{tabular}{|c|c|c|c|c|}
\hline Especie & $\begin{array}{l}\text { Densidad } \\
\text { Relativa }\end{array}$ & $\begin{array}{c}\text { Cobertura } \\
\text { Relativa }\end{array}$ & $\begin{array}{c}\text { Frecuencia } \\
\text { Relativa }\end{array}$ & $\begin{array}{c}\text { Valor de } \\
\text { Importancia }\end{array}$ \\
\hline \multicolumn{5}{|l|}{ Estrato herbáceo } \\
\hline Selaginella rupincola Underw. & 31.068 & 17.504 & 4.000 & 52.571 \\
\hline Schkuhria pinnata (Lam.) Kuntze & 16.993 & 25.339 & 4.000 & 46.332 \\
\hline Tagetes micrantha Cav. & 8.067 & 8.613 & 3.000 & 19.680 \\
\hline Cheilanthes bonariensis (Willd.) Proctor & 9.269 & 5.131 & 1.000 & 15.400 \\
\hline Heterosperma pinnatum Cav. & 3.948 & 5.230 & 4.000 & 13.178 \\
\hline Cosmos parviflorus (Jacq.) Pers. & 4.291 & 3.560 & 4.000 & 11.851 \\
\hline Tagetes Iunulata Ortega & 2.660 & 1.699 & 4.000 & 8.360 \\
\hline Cyperus seslerioides Kunth & 2.575 & 2.367 & 2.000 & 6.942 \\
\hline Tillandsia fresnilloensis W. Weber \& Ehlers & 1.716 & 1.747 & 3.000 & 6.464 \\
\hline Arenaria lycopodioides Willd. ex Schltdl. & 1.716 & 2.542 & 2.000 & 6.258 \\
\hline Plantago linearis Kunth & 1.287 & 2.051 & 2.000 & 5.338 \\
\hline Commelina dianthifolia Delile & 1.116 & 1.199 & 3.000 & 5.315 \\
\hline Crusea diversifolia (Kunth) Anderson & 1.888 & 0.888 & 2.000 & 4.776 \\
\hline Tridax balbisioides (Kunth) A. Gray & 1.202 & 1.336 & 2.000 & 4.538 \\
\hline Polypodium thyssanolepis $\mathrm{A}$. Br. ex Klotzsch & 2.146 & 1.009 & 1.000 & 4.155 \\
\hline Muhlenbergia rigida (Kunth) Trin. & 0.343 & 0.451 & 3.000 & 3.794 \\
\hline Helianthemum glomeratum Lag. & 0.601 & 0.668 & 2.000 & 3.269 \\
\hline Desmodium neomexicanum A. Gray & 0.687 & 0.516 & 2.000 & 3.202 \\
\hline $\begin{array}{l}\text { Sisyrinchium tenuifolium Humb. \& Bonpl. } \\
\text { ex Willd. }\end{array}$ & 0.429 & 0.684 & 2.000 & 3.113 \\
\hline Echinocereus polyacanthus Engelm. & 0.515 & 0.551 & 2.000 & 3.066 \\
\hline $\begin{array}{l}\text { Psacalium sinuatum (Cerv.) } \\
\text { H. Rob. \& Brettell }\end{array}$ & 0.343 & 0.547 & 2.000 & 2.890 \\
\hline Oxalis alpina (Rose) Knuth & 1.202 & 0.565 & 1.000 & 2.767 \\
\hline Stevia lucida Lag. & 0.429 & 0.215 & 2.000 & 2.644 \\
\hline Galinsoga parviflora Cav. & 0.172 & 0.273 & 2.000 & 2.445 \\
\hline Allium glandulosum Link \& Otto & 0.515 & 0.820 & 1.000 & 2.335 \\
\hline Mammilaria gummifera Engelm. & 0.172 & 0.094 & 2.000 & 2.266 \\
\hline
\end{tabular}

Matorral de Mimosa-Opuntia. Ocupa algunas laderas riolíticas ubicadas al oriente del área de estudio y se encuentra también en manchones dispersos en suelos erosionados sobre substratos de rocas sedimentarias conglomeradas. En el parque cubre una superficie de 78.19 ha. La altura de esta comunidad es de 1 a $1.5 \mathrm{~m}$ y está compuesta principalmente de Mimosa aculeaticarpa, Lippia durangensis, Jatropha dioica y Opuntia durangensis. En algunas superficies se observa asociado a Pinus cembroides. En el estrato herbáceo son importantes Heterosperma pinnatum, Tridax balbisioides, Tagetes lunulata, Schkuhria pinnata, Gomphrena serrata, Tagetes micrantha, Desmodium neomexicanum, Bouteloua gracilis, Aristida adscensionis, Euphorbia hirta, Eragrostis intermedia, Evolvulus 
alsinoides, Sanvitalia procumbens, Portulaca pilosa, Zornia thymifolia, Selaginella rupincola, Galinsoga parviflora, Dichondra argentea y Sisyrinchium tenuifolium (Cuadro 7).

Cuadro 7. Índices relativos de densidad, cobertura, frecuencia y valor de importancia de los componentes del matorral de Mimosa-Opuntia de la Sierra de Órganos, Sombrerete, Zacatecas.

Especie

Estrato arbóreo

Pinus cembroides Zucc.

Quercus grisea Liebm.

Quercus laeta Liebm.

Yucca decipiens Trel.

Estrato arbustivo y subarbustivo

Mimosa aculeaticarpa Ortega

Lippia durangensis Mold.

Opuntia durangensis Britton \& Rose

Jatropha dioica Sessé ex Cerv.

Opuntia robusta $\mathrm{H}$. Wendl.

Eysenhardtia polystachya (Ortega) Sarg.

Bouvardia scabrida Mart. \& Gal.

Mimosa dysocarpa Benth.

Trixis angustifolia DC.

Forestiera durangensis Standl.

Montanoa leucantha

(Lag. \& Segura) S. F. Blake

Dalea bicolor Humb. \& Bonpl. ex Willd.

Ageratum corymbosum Zuccagni ex Pers.

Loeselia mexicana (Lam.) Brand

Stevia salicifolia Cav.

Juniperus deppeana Steud.

Quercus potosina Trel.

Arctostaphylos pungens Kunth

Estrato herbáceo

Heterosperma pinnatum Cav.

Tridax balbisioides (Kunth) A. Gray

Tagetes lunulata Ortega
Densidad Cobertura Frecuencia Valor de

Relativa Relativa Relativa Importancia

$\begin{array}{llll}0.018 & 5.978 & 1.563 & 7.559 \\ 0.002 & 1.931 & 0.781 & 2.715 \\ 0.005 & 0.462 & 0.781 & 1.247 \\ 0.002 & 0.028 & 0.781 & 0.812\end{array}$

$\begin{array}{llll}0.382 & 34.232 & 2.344 & 36.957\end{array}$

$\begin{array}{llll}0.039 & 3.838 & 1.563 & 5.439\end{array}$

$\begin{array}{llll}0.036 & 3.348 & 1.563 & 4.946\end{array}$

$\begin{array}{llll}1.363 & 0.535 & 1.563 & 3.460\end{array}$

$\begin{array}{llll}0.014 & 0.467 & 2.344 & 2.824\end{array}$

$\begin{array}{llll}0.020 & 0.786 & 1.563 & 2.369\end{array}$

$\begin{array}{llll}0.136 & 0.047 & 1.563 & 1.746\end{array}$

$\begin{array}{llll}0.011 & 0.605 & 0.781 & 1.398\end{array}$

$\begin{array}{llll}0.048 & 0.338 & 0.781 & 1.167\end{array}$

$\begin{array}{llll}0.011 & 0.355 & 0.781 & 1.148\end{array}$

$\begin{array}{llll}0.136 & 0.107 & 0.781 & 1.024\end{array}$

$\begin{array}{llll}0.091 & 0.040 & 0.781 & 0.912\end{array}$

$\begin{array}{llll}0.091 & 0.015 & 0.781 & 0.887\end{array}$

$\begin{array}{llll}0.045 & 0.036 & 0.781 & 0.862\end{array}$

$\begin{array}{llll}0.045 & 0.020 & 0.781 & 0.847\end{array}$

$\begin{array}{llll}0.002 & 0.057 & 0.781 & 0.841\end{array}$

$\begin{array}{llll}0.002 & 0.047 & 0.781 & 0.830\end{array}$

$\begin{array}{llll}0.002 & 0.037 & 0.781 & 0.820\end{array}$ 
Cuadro 7. Continuación.

\begin{tabular}{|c|c|c|c|c|}
\hline Especie & $\begin{array}{l}\text { Densidad } \\
\text { Relativa }\end{array}$ & $\begin{array}{l}\text { Cobertura } \\
\text { Relativa }\end{array}$ & $\begin{array}{c}\text { Frecuencia } \\
\text { Relativa }\end{array}$ & $\begin{array}{c}\text { Valor de } \\
\text { Importancia }\end{array}$ \\
\hline Schkuhria pinnata (Lam.) Kuntze & 6.179 & 1.562 & 2.344 & 10.085 \\
\hline Gomphrena serrata L & 2.590 & 1.187 & 2.344 & 6.120 \\
\hline Tagetes micrantha Cav. & 2.544 & 1.058 & 2.344 & 5.946 \\
\hline Desmodium neomexicanum A. Gray & 2.181 & 0.765 & 2.344 & 5.289 \\
\hline Bouteloua gracilis (Kunth) Lag. & 1.999 & 0.837 & 2.344 & 5.180 \\
\hline Aristida adscensionis L. & 1.454 & 0.531 & 2.344 & 4.328 \\
\hline Euphorbia hirta L. & 1.272 & 0.544 & 2.344 & 4.160 \\
\hline Eragrostis intermedia Hitchc. & 1.181 & 0.571 & 2.344 & 4.096 \\
\hline Evolvulus alsinoides L. & 1.590 & 0.740 & 1.563 & 3.893 \\
\hline $\begin{array}{l}\text { Sanvitalia angustifolia Engelm. } \\
\text { ex A. Gray }\end{array}$ & 2.090 & 0.349 & 0.781 & 3.221 \\
\hline Portulaca pilosa L. & 0.909 & 0.695 & 1.563 & 3.166 \\
\hline Zornia thymifolia Kunth & 0.909 & 0.539 & 1.563 & 3.010 \\
\hline Selaginella rupincola Underw. & 0.409 & 0.248 & 2.344 & 3.001 \\
\hline Galinsoga parviflora Cav. & 0.818 & 0.593 & 1.563 & 2.973 \\
\hline $\begin{array}{l}\text { Dichondra argentea Humb. \& Bonpl. } \\
\text { ex Willd. }\end{array}$ & 0.772 & 0.464 & 1.563 & 2.798 \\
\hline
\end{tabular}

Pastizal. Este tipo de vegetación se encuentra sobre suelos aluviales y rocas sedimentarias conglomeradas en las áreas planas y abiertas y en las laderas que marcan los límites orientales del área de estudio. En el parque cubre una superficie de 156.97 ha.

Es una comunidad dominada por plantas herbáceas en la que predominan especies de compuestas y de gramíneas. Se compone principalmente de Heterosperma pinnatum, Pectis prostrata, Bouteloua gracilis, Euphorbia hirta, Melampodium sericeum, Eragrostis intermedia, Crusea diversifolia, Schkuhria pinnata, Microchloa kunthii, Dichondra argentea y Aristida adscensionis. Dentro de la superficie que ocupa esta comunidad, existe en algunas áreas cercanas a las laderas de los cerros un matorral asociado con el pastizal compuesto por Mimosa aculeaticarpa y Acacia schaffneri, posiblemente por influencia del sobrepastoreo. En el pastizal ubicado en las laderas en el oriente del parque se encuentran frecuentemente cactáceas como Mammillaria gummifera, Opuntia durangensis, 0 . robusta y Stenocactus zacatecasensis (Cuadro 8).

Vegetación acuática y subacuática. Todos los arroyos del área de estudio son temporales, por lo que no cuentan con vegetación estrictamente acuática. La única comunidad de plantas acuáticas en el parque se localiza en los alrededores de un manantial y un presón adyacente, a $2300 \mathrm{~m}$ de altitud, en terreno plano rodeado de pastizal. El manantial tiene una baja escorrentía de agua, y el presón 
Cuadro 8. Índices relativos de densidad, cobertura, frecuencia y valor de importancia de los componentes del pastizal de la sierra de Órganos, Sombrerete, Zacatecas.

$\begin{array}{cc}\text { Especie } & \text { Densidad Cobertura Frecuencia Valor de } \\ \text { Relativa } & \text { Relativa Relativa Importancia }\end{array}$

Estrato arbustivo y subarbustivo

$\begin{array}{llllr}\text { Mimosa aculeaticarpa Ortega } & 0.040 & 9.248 & 2.857 & 12.145 \\ \begin{array}{l}\text { Acacia schaffneri (S. Watson) } \\ \quad \text { F. J. Herm. }\end{array} & 0.007 & 5.208 & 0.952 & 6.168 \\ \begin{array}{l}\text { Juniperus deppeana Steud. } \\ \text { Opuntia durangensis Britton \& Rose }\end{array} & 0.006 & 3.194 & 1.905 & 5.104 \\ \text { Opuntia robusta H. Wendl. } & 0.023 & 0.688 & 1.905 & 2.615 \\ \text { Calliandra humilis (Schltdl.) Benth. } & 0.004 & 0.127 & 1.905 & 2.036 \\ \text { Mimosa dysocarpa Benth. } & 0.284 & 0.365 & 0.952 & 1.601 \\ \text { Clematis drummondii Torr. \& A. Gray } & 0.001 & 0.223 & 0.952 & 1.177 \\ & 0.028 & 0.036 & 0.952 & 1.017\end{array}$

Estrato herbáceo

$\begin{array}{lrrrr}\text { Heterosperma pinnatum Cav. } & 25.039 & 19.770 & 2.857 & 47.667 \\ \text { Pectis prostrata Cav. } & 17.184 & 11.261 & 1.905 & 30.350 \\ \text { Bouteloua gracilis (Kunth) Lag. } & 10.407 & 8.134 & 2.857 & 21.398 \\ \text { Euphorbia hirta L. } & 5.671 & 4.410 & 2.857 & 12.938 \\ \text { Melampodium sericeum Lag. } & 4.537 & 4.450 & 1.905 & 10.892 \\ \text { Eragrostis intermedia Hitchc. } & 4.962 & 3.935 & 1.905 & 10.802 \\ \text { Crusea diversifolia (Kunth) Anderson } & 3.289 & 3.514 & 2.857 & 9.661 \\ \text { Schkuhria pinnata (Lam.) Kuntze } & 3.233 & 3.197 & 2.857 & 9.287 \\ \text { Microchloa kunthii Desv. } & 4.565 & 3.641 & 0.952 & 9.159 \\ \text { Dichondra argentea Humb. \& Bonpl. } & 2.297 & 2.288 & 2.857 & 7.442 \\ \quad \text { ex Willd. } & & & & \\ \text { Aristida adscensionis L. } & 2.807 & 2.391 & 1.905 & 7.104 \\ \text { Arenaria lycopodioides Willd. ex Schltdl. } & 2.013 & 2.019 & 2.857 & 6.889 \\ \text { Gomphrena serrata L. } & 1.560 & 1.177 & 2.857 & 5.593 \\ \text { Richardia tricocca (Torr. \& A. Gray) Standl. } & 0.737 & 0.884 & 2.857 & 4.479 \\ \text { Sida linearis Cav. } & 0.567 & 0.679 & 2.857 & 4.104 \\ \text { Selaginella rupincola Underw. } & 1.730 & 1.380 & 0.952 & 4.062 \\ \text { Oxalis corniculata L. } & 0.454 & 0.397 & 2.857 & 3.708 \\ \text { Evolvulus alsinioides L. } & 0.822 & 0.652 & 1.905 & 3.379 \\ \text { Erodium cicutarium (L.) L'Hér. ex Aiton } & 1.333 & 0.862 & 0.952 & 3.147 \\ \text { Dyssodia papposa (Vent.) Hitchc. } & 0.510 & 0.643 & 1.905 & 3.058 \\ \text { Guilleminea densa (Willd.) Moq. } & 0.567 & 0.403 & 1.905 & 2.875 \\ \text { Portulaca pilosa L. } & 0.454 & 0.493 & 1.905 & 2.851 \\ \text { Polygala rivinifolia Kunth } & 0.425 & 0.335 & 1.905 & 2.665 \\ \text { Lycurus phleoides Kunth } & 0.284 & 0.309 & 1.905 & 2.498 \\ \text { Sisyrinchium tenuifolium Humb. \& Bonpl. } & 0.312 & 0.215 & 1.905 & 2.431 \\ \quad \text { ex Willd. } & & & & \\ \text { Zornia thymifolia Kunth } & 0.227 & 0.237 & 1.905 & 2.369\end{array}$


Cuadro 8. Continuación.

\begin{tabular}{lcccc}
\hline Especie & $\begin{array}{c}\text { Densidad } \\
\text { Relativa }\end{array}$ & $\begin{array}{c}\text { Cobertura } \\
\text { Relativa }\end{array}$ & $\begin{array}{c}\text { Frecuencia } \\
\text { Relativa }\end{array}$ & $\begin{array}{c}\text { Valor de } \\
\text { Importancia }\end{array}$ \\
Plantago linearis Kunth & 0.198 & 0.228 & 1.905 & 2.331 \\
Brickellia vernicosa B. L. Rob. & 0.170 & 0.128 & 1.905 & 2.203 \\
Portulaca oleracea L. & 0.113 & 0.078 & 1.905 & 2.096 \\
Aspicarpa hirtella A. Gray & 0.057 & 0.059 & 1.905 & 2.021 \\
Tagetes micrantha Cav. & 0.482 & 0.384 & 0.952 & 1.819 \\
Bouteloua curtipendula (Michx.) & 0.425 & 0.339 & 0.952 & 1.717 \\
G. S. Torr. & & & & \\
Tridax balbisioides (Kunth) A. Gray & 0.425 & 0.339 & 0.952 & 1.717 \\
Muhlenbergia rigida (Kunth) Trin. & 0.340 & 0.271 & 0.952 & 1.564 \\
& & & & \\
\hline
\end{tabular}

mide aproximadamente $10 \mathrm{~m}$ de ancho y $15 \mathrm{~m}$ de largo. Se encuentran a unos $800 \mathrm{~m}$ al oeste de la entrada del parque, cercanos al arroyo Tanquecitos.

Las principales especies encontradas son: Lemna gibba (flotante), Heteranthera peduncularis e Hydrocotyle ranunculoides (arraigadas con hojas flotantes), y Eleocharis sp., Mimulus glabratus y Ranunculus hydrocharoides (arraigadas emergentes). Entre las plantas subacuáticas que se desarrollan en las orillas del cuerpo de agua destacan Cyperus niger, Polygonum hydropiperoides y Sisyrinchium convolutum.

Debido a que el presón es usado como abrevadero para ganado, la vegetación de sus alrededores presenta un alto grado de perturbación. En esos sitios son abundantes plantas como Aristida adscensionis, Alternanthera caracassana, Amaranthus hybridus, Chenopodium graveolens, Heterosperma pinnatum, Salsola tragus y Schkuhria pinnata.

La superficie de cultivo del área de estudio invade parte del territorio del parque. Este terreno tiene una superficie de 26.46 ha y está representado en el Apéndice 2.

Similitud entre comunidades

La relación florística entre las nueve comunidades reconocidas fue evaluada mediante la comparación de los coeficientes de similitud de Jaccard. En el Cuadro 9 se muestran los valores respectivos calculados. La mayor afinidad se presenta entre el bosque de Pinus y el matorral de Mimosa-Opuntia (41\%). La vegetación acuática no tiene ninguna especie en común con el resto de las comunidades (índice $0)$. Otras agrupaciones con escasa relación con las demás son el bosque de PinusJuniperus con la vegetación de arroyos pedregosos y con la vegetación de peñascos (10\%), la vegetación de peñascos con el pastizal (15\%) y el pastizal con el bosque de Pinus-Juniperus y con la vegetación de arroyos pedregosos (17\%) (Cuadro 9). 
Cuadro 9. Valores del índice de similitud de Jaccard obtenidos entre las comunidades. $\mathrm{BQ}=$ Bosque de Quercus; $\mathrm{BP}=$ Bosque de Pinus; $\mathrm{BPQ}=$ Bosque de Pinus-Quercus; BPJ = Bosque de Pinus-Juniperus; VAP = Vegetación de arroyos pedregosos; VP = Vegetación de peñascos; $\mathrm{MMO}=$ Matorral de Mimosa-Opuntia; $\mathrm{P}=$ Pastizal, y $\mathrm{VA}=$ Vegetación acuática y subacuática. Los valores en la diagonal representan la riqueza florística de cada comunidad.

\begin{tabular}{|l|c|c|c|c|c|c|c|c|c|}
\hline & $\mathrm{BQ}$ & $\mathrm{BP}$ & $\mathrm{BPQ}$ & $\mathrm{BPJ}$ & $\mathrm{VAP}$ & $\mathrm{VP}$ & $\mathrm{MMO}$ & $\mathrm{P}$ & $\mathrm{VA}$ \\
\hline $\mathrm{BQ}$ & 87 & $34 \%$ & $25 \%$ & $25 \%$ & $19 \%$ & $23 \%$ & $38 \%$ & $24 \%$ & $0 \%$ \\
\hline $\mathrm{BP}$ & & 88 & $30 \%$ & $24 \%$ & $18 \%$ & $26 \%$ & $41 \%$ & $29 \%$ & $0 \%$ \\
\hline $\mathrm{BPQ}$ & & & 231 & $20 \%$ & $36 \%$ & $20 \%$ & $33 \%$ & $31 \%$ & $0 \%$ \\
\hline BPJ & & & & 90 & $10 \%$ & $10 \%$ & $20 \%$ & $17 \%$ & $0 \%$ \\
\hline VAP & & & & & 144 & $20 \%$ & $18 \%$ & $17 \%$ & $0 \%$ \\
\hline VP & & & & & & 58 & $28 \%$ & $15 \%$ & $0 \%$ \\
\hline MMO & & & & & & & 106 & $32 \%$ & $0 \%$ \\
\hline P & & & & & & & & 176 & $0 \%$ \\
\hline VA & & & & & & & & & 11 \\
\hline
\end{tabular}

Con el fin de obtener una representación visual de la relación florística entre las comunidades se generó un fenograma (Fig. 2). Se aprecia que la mayor afinidad encontrada es entre el bosque de Pinus y el bosque de Pinus-Quercus (48\%) y de estos dos con el matorral de Mimosa-Opuntia. La comunidad con menor semejanza florística con el resto es la vegetación acuática y subacuática $(0 \%)$, seguida por la vegetación de arroyos pedregosos y la de peñascos, con $33 \%$ de semejanza entre sí, pero con únicamente $24 \%$ en relación con el resto de las comunidades del área.

Las discrepancias observadas entre los valores de la matriz de similitud (Cuadro 9) y los del fenograma (Fig. 2) provienen del algoritmo usado (UPGMA) en la construcción de este último. Con este algoritmo se calculan nuevos índices 
a partir de la matriz de similitud y la media aritmética extraída de la similitud de un núcleo de dos entidades vegetales con respecto a las demás comunidades, derivando una matriz con cuyos datos se establecen los agrupamientos en el fenograma.

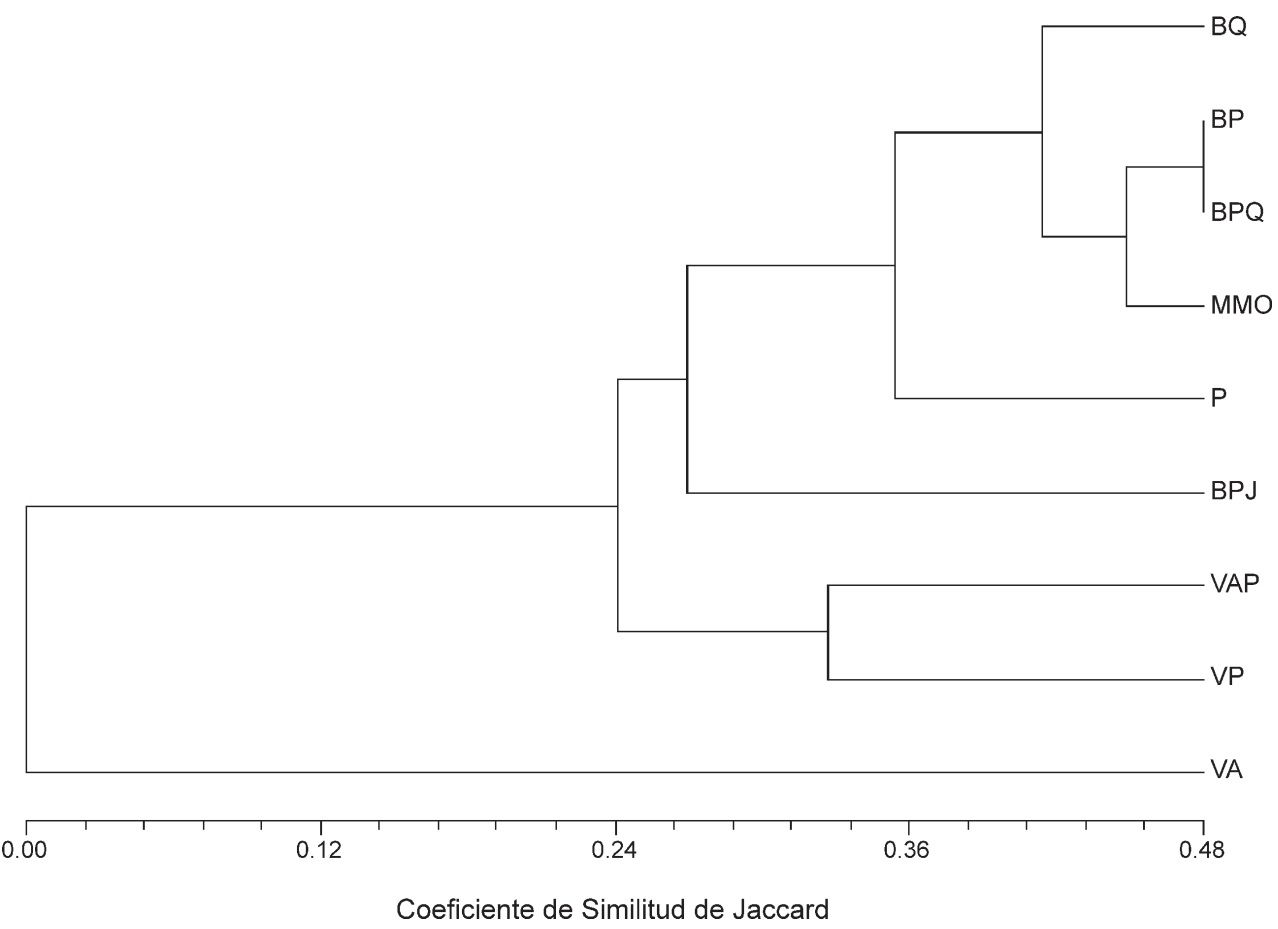

Fig. 2. Fenograma de similitud florísitica entre nueve tipos de vegetación de la Sierra de Órganos. $\mathrm{BQ}=$ Bosque de Quercus, $\mathrm{MMO}=$ Matorral de Mimosa-Opuntia, $\mathrm{BP}=$ Bosque de Pinus, $\mathrm{P}=$ Pastizal, $\mathrm{BPQ}=$ Bosque de Pinus-Quercus, VAP = Vegetación de arroyos pedregosos, VP = Vegetación de peñascos, BPJ = Bosque de Pinus-Juniperus y VA = Vegetación acuática y subacuática.

\section{DISCUSIÓN}

Las formaciones columnares y las grandes rocas riolíticas y conglomerados del área de estudio sugieren un desarrollo geológico reciente de movimiento de la corteza terrestre que ocasionó una rápida erosión (Shannon y Kramer, 1973). Este proceso erosivo determinó una heterogeneidad de geoformas, de condiciones de los suelos y de variantes microclimáticas que afectan fuertemente los patrones de 
distribución de las plantas y de sus comunidades. La vegetación prevaleciente en la zona está representada por agrupaciones con dominancia fisonómica de Pinus cembroides, en colindancia con encinares, matorrales xerófilos y pastizales. La comparación de la repartición actual de la cubierta vegetal y la que revelan las fotografías de hace 33 años, muestra que esta distribución no difiere de manera significativa, lo que parece indicar que desde que las imagenes fueron tomadas la agricultura se ha desarrollado principalmente fuera de los límites del parque. Por otro lado, en la actualidad es muy evidente el impacto que la vegetación está sufriendo debido al pastoreo y al ramoneo de ganado. La existencia de matorrales de Mimosa-Opuntia en el área de estudio es, por lo menos en parte, producto del sobrepastoreo ejercido en el pastizal, aspecto que ha sido estudiado por Brown (1982) en otras áreas del país.

El bosque de Pinus y el bosque de Pinus-Quercus revelan una relativamente alta semejanza florística (48\%) y aparecen unidos en el fenograma de la Fig. 2. Aunque indudablemente representan variantes de un mismo tipo de vegetación, se consideran como unidades independientes debido a que su grado de similitud no supera $50 \%$, considerado como umbral para agrupar comunidades (Barbour et al., 1987) y menos aún $62.5 \%$ propuesto por Hagmeier y Stults (1964), ó 66.6\% propuesto por Sánchez y López (1988) como valor crítico para la separación de floras y faunas. Por otra parte, estas entidades se consideran como unidades independientes con base en el criterio fisonómico-florístico aplicado en este trabajo para definir las unidades de vegetación, ya que las diferencias fisonómicas entre ambas son perceptibles incluso en las fotografías aéreas.

La flora más singular es la de la vegetación acuática y subacuática, que no comparte ninguna especie con el resto de las formaciones vegetales del área. La comunidad propia de peñascos presenta también una escasa relación florística con el resto, debido a la especialización rupícola de muchos de sus componentes.

Otras agrupaciones que albergan especies de distribución local restringida son la vegetación de arroyos pedregosos y el bosque de Pinus-Juniperus, única formación vegetal del área que se desarrolla sobre substrato de roca caliza. Este bosque presenta una composición de especies muy diferente de la de otros pinares del área, que se desarrollan sobre suelos derivados de riolitas. Tal hecho coincide con lo señalado por Rzedowski (1978) acerca de que los pinares tienen preferencia por los suelos derivados de roca volcánica y se les encuentra también sobre suelos provenientes de roca caliza.

En la Sierra de Órganos las comunidades de pastizal y matorral de MimosaOpuntia se distribuyen principalmente sobre conglomerados cubiertos por xerosoles, suelos característicos de superficies planas o con pendientes ligeras de alta exposición a la insolación. Los demás tipos de vegetación del área se ubican principalmente sobre litosoles.

El bosque de Quercus se localiza en las laderas ubicadas al oriente y en las mesas de algunos cerros con menor humedad, como el de La Peña Larga. Sus árboles son de hoja pequeña, adaptados a la escasa disponibilidad de agua, lo que coincide con lo señalado por Rzedowski (1978) para otras partes de México. En 
el bosque de Pinus-Quercus los encinos se concentran en las partes altas de las laderas, donde la tierra es más escasa y es mayor la pedregosidad y por lo tanto la disponibilidad de humedad y nutrientes probablemente es más pobre. Por el contrario, los pinos se encuentran en mayor densidad donde los suelos son más profundos, sobre todo en los valles intermontanos.

La vegetación de peñascos que se desarrolla sobre riolitas presenta singularidades notorias por la baja disponibilidad de humedad, por lo que varias especies tienen adaptaciones que les permiten utilizar el agua de escurrimiento de las rocas, como Tillandsia fresnilloensis y Selaginella rupincola. La cubierta vegetal de arroyos pedregosos, como la encontrada en el arroyo El Salto, debe su peculiaridad a la abundancia de fragmentos rocosos en su lecho, así como a la mayor humedad y a condiciones de menor luminosidad.

Algunas especies se encuentran restringidas a condiciones específicas de substratos, por ejemplo: Begonia gracilis, Coreopsis macvaughii, Erythrina montana, Hypericum silenoides, Juncus acuminatus, J. arcticus, Karinia mexicana, Lobelia laxiflora, Prochnyanthes mexicana y Silene laciniata, las cuales se localizan sobre los arroyos pedregosos. Coreopsis rudis, Eutetras sp. Mammillaria moelleriana, Sedum glabrum, Stenocactus zacatecasensis y Tillandsia fresnilloensis se distribuyen esencialmente sobre la zona de peñascos. A su vez Carex planostachys, Ceanothus greggii var. lanuginosus, Rhus aromatica var. trilobata, Rhus virens, Salvia greggii, Sarcostemma cf. torreyi y Schoenocaulon texanum se encontraron sólamente en el área de roca caliza.

Tres especies se encuentran en condiciones ambientales muy frágiles, que de ser alteradas pueden ocasionar su desaparición. Coreopsis macvaughii restringe su población a una colonia formada por unos cuantos individuos que crecen sobre una pared rocosa a un lado de una cascada intermitente; Eutetras sp. se desarrolla en algunas ranuras de las paredes rocosas y su población es muy dispersa y escasa; y Tillandsia fresnilloensis, que crece esencialmente sobre las paredes rocosas, es endémica de Zacatecas con distribución restringida a Sierra de Órganos y el municipio de Fresnillo (Weber, 1983). Las prácticas de rapeleo y de escalamiento que se llevan a cabo en el parque ponen en riesgo a estas especies.

La agrupación de los valores de importancia en categorías permite visualizar la dominancia de cada especie en las comunidades vegetales y en el área de estudio en general. Con la suma de estos valores para cada especie, y por cada tipo de vegetación donde ésta se encuentra, se obtiene el valor de importancia acumulado (Apéndice 1). Con base en estos datos se precisa que Heterosperma pinnatum, Schkuhria pinnata, Tagetes micrantha, Euphorbia hirta, Pinus cembroides, Mimosa aculeaticarpa y Aristida adscensionis, son las especies más sobresalientes en toda el área porque alcanzan valores acumulados desde 24 hasta 32. Heterosperma pinnatum es, con mucho, el elemento dominante en el área de pastizal y en el matorral de Mimosa-Opuntia, reflejando posibles efectos de sobrepastoreo. En contraste, especies como Adenophyllum porophylum, Aegopogon tenellus, Ageratina rubricaulis, Ageratum corymbosum, Bidens ferulifolia y Buddleja scordioides, entre otros, sólo alcanzan un valor acumulado de 1. 
En la mayor parte de las comunidades vegetales descritas los valores de importancia de algunas especies herbáceas son superiores a los de las especies arbóreas. Esto es reflejo de la circunstancia de que las comunidades del área son abiertas, donde los árboles se presentan como elementos muy espaciados sobre una densa cubierta herbácea.

\section{CONCLUSIONES}

Este trabajo constituye la primera aportación al conocimiento de la flora y la vegetación de la Sierra de Órganos y podrá servir de base para monitoreos futuros en el recién decretado Parque Nacional.

La vegetación de peñascos y la de arroyos pedregosos contienen especies en riesgo, para las que es necesario tomar medidas especiales de conservación dado el uso turístico del parque.

La información recabada sobre la distribución y el grado de abundancia de las especies de plantas de la Sierra de Órganos constituye una herramienta para la elaboración de planes de conservación de esa flora.

\section{AGRADECIMIENTOS}

Se agradece al CONACYT por su apoyo mediante el otorgamiento de una beca de postgrado para el primer autor. A Miguel Adame G. por su apoyo y compañía en el trabajo de campo; a José Luis Villaseñor, Raquel Galván, Fernando Zavala, Salvador Arias M., J. Jesús Balleza C., Adolfo Espejo, Martha González, Yolanda Herrera y Paul M. Peterson por su apoyo en la identificación de material de herbario; a Heike Vibrans por su ayuda en el estudio de la vegetación; a Jorge A. Tena por su asesoría en el análisis de similitud; a René Ruiz G. y a Carlos Ledezma M. por su apoyo en la elaboración del mapa de vegetación, y a Enrique Ortiz B., Marco A. Márquez y A. Cortés O. por su ayuda para cuantificar la superficie de los tipos de vegetación. El Dr. J. Rzedowski y tres revisores anónimos aportaron sugerencias que nos permitieron incrementar la calidad del manuscrito. Los encargados de los herbarios CHAPA, CIIDIR, HUAZ y MEXU brindaron facilidades para la consulta de sus colecciones.

\section{LITERATURA CITADA}

Aldrete, M. E. 1981. Estudio ecológico de los agostaderos del noreste del estado de Zacatecas. Tesis de Licenciatura. Universidad Autónoma Chapingo, Departamento de Zootecnia. Chapingo, México. 285 pp.

Anónimo. 1971. Carta edafológica. F-13-B-14. Escala 1:50 000. Comisión de Estudios del Territorio Nacional. Secretaría de Programación y Presupuesto. México, D.F. 
Anónimo. 1979. Carta geológica F-13-B-14. Escala 1:50 000. Comisión de Estudios del Territorio Nacional. Secretaría de Programación y Presupuesto. México, D.F.

Anónimo. 1980a. Coeficientes de agostadero de la República Mexicana. Estados de Zacatecas y Aguascalientes. Secretaría de Agricultura y Recursos Hidráulicos, Comisión Técnico Consultiva para la Determinación de los Coeficientes de Agostadero. México, D.F. 267 pp.

Anónimo. 1980b. Estado de Zacatecas. Carta climatológica. Escala 1: 1000 000. Dirección General de Geografía del Territorio Nacional. Secretaría de Programación y Presupuesto. México, D.F.

Anónimo. 1980c. Estado de Zacatecas. Carta geológica. Dirección General de Geografía del Territorio Nacional. Secretaría de Programación y Presupuesto. México, D.F.

Anónimo. 1980d. Estado de Zacatecas. Carta de regionalización fisiográfica. Dirección General de Geografía del Territorio Nacional. Secretaría de Programación y Presupuesto. México, D.F.

Anónimo. 1981. Síntesis geográfica de Zacatecas. Dirección General de Geografía del Territorio Nacional. Secretaría de Programación y Presupuesto. México, D.F.

Balleza C., J. J. 1992. Gramíneas del estado de Zacatecas. Tesis de Maestría en Ciencias, Colegio de Postgraduados. Chapingo, México. 108 pp.

Balleza C., J. J. y J. L. Villaseñor. 2002. La familia Asteraceae en el estado de Zacatecas (México). Acta Bot. Mex. 59: 5-69.

Barbour, M. G., J. H. Burk y W. D. Pitts. 1987.Terrestrial plant ecology. 2a. ed. The Benjamin/ Cummings Publishing Company. Menlo Park, California. 634 pp.

Brown, D. E. 1982. Semidesert grassland. In: Brown, D. E. (ed.). Desert plants (Special issue: biotic communities of the American Southwest - United States and Mexico) 4(1-4): 123-131.

Claverán, A. R. 1961. Notas sobre las zonas ganaderas del estado de Zacatecas. Escuela Nacional de Agricultura. Tesis de Licenciatura. Chapingo, México. 153 pp.

Cronquist, A. 1988. The evolution and classification of flowering plants. 2a ed. New York Botanical Garden. Bronx, Nueva York. 555 pp.

Curtis, J. T. y R. P. Mclntosh. 1951. An upland forest continuum in the prairie-forest border region of Wisconsin. Ecology 32: 476-496.

Dávila, P. y V. Sosa. 1994. El conocimiento florístico de México. Bol. Soc. Bot. México 55: 21-27.

Enríquez E., E. D. 1998. Estudio florístico del cerro La Cantarilla, municipio de Moyahua, estado de Zacatecas, México. Tesis de Maestría en Ciencias. Colegio de Postgraduados. Montecillo, Texcoco, México. 86 pp.

Ferrusquía-Villafranca, I. 1993. Geology of Mexico: a synopsis. In: Ramamoorty, T. P., R. Bye, A. Lot y J. Fa (eds.). Biological diversity of Mexico: origins and distribution. Oxford University Press. Nueva York. pp. 3-107.

González, A. J. 1998. Los bosques piñoneros de México: estudio del bosque de Pinus johannis M. F. Robert en Concepción del Oro, Zacatecas. Tesis de Licenciatura. Universidad Autónoma Chapingo. División de Ciencias Forestales. Chapingo, México. 179 pp.

González E., M. 1975. Distribución espacial de la vegetación y su interpretación sucesional en el noreste del estado de Zacatecas. Tesis de Licenciatura. Escuela Nacional de Agricultura. Chapingo, México. 263 pp.

González-Elizondo, S., M. González-Elizondo y A. Cortés-Ortiz. 1993. Vegetación de la reserva de la biosfera "La Michilía", Durango, México. Acta Bot. Mex. 22: 1-104. 
Guzmán H., G. y L. Vela G. 1960. Contribución al conocimiento de la vegetación del suroeste del estado de Zacatecas. Bol. Soc. Bot. México. 25: 40-60.

Hagmeier, E. M. y C. D. Stults. 1964. A numerical analysis of the distribution patterns of North American mammals. Syst. Zool. 13(3): 125-155.

Luna C., M., E. García M. y B. Vázquez H. 1997. Cambios en la composición botánica de dos agostaderos de Zacatecas, México en exclusión y agostadero. Agrociencia 31(3): 313-321.

Magurran, A. E. 1988. Ecological diversity and its measurement. Princeton University Press. Princeton, Nueva Jersey. 179 pp.

McVaugh, R. 1983. Flora Novo-Galiciana 14. Gramineae. University of Michigan Press. Ann Arbor, Michigan. 436 pp.

McVaugh, R. 1984. Flora Novo-Galiciana 12. Compositae. University of Michigan Press. Ann Arbor, Michigan. $1157 \mathrm{pp}$.

McVaugh, R. 1985. Flora Novo-Galiciana 16. Orchidaceae. University of Michigan Press. Ann Arbor, Michigan. 363 pp.

McVaugh, R. 1987. Flora Novo-Galiciana 5. Leguminosae. University of Michigan Press. Ann Arbor, Michigan. 786 pp.

McVaugh, R. 1989. Flora Novo-Galiciana 15. Bromeliaceae to Dioscoreaceae. University of Michigan Herbarium. Ann Arbor, Michigan. 398 pp.

McVaugh, R. 1992. Flora Novo-Galiciana 17. Gymnosperms and Pteridophytes. University of Michigan Herbarium. Ann Arbor, Michigan. 467 pp.

McVaugh, R. 1993. Flora Novo-Galiciana 13. Limnocharitaceae to Typhaceae. University of Michigan Herbarium. Ann Arbor, Michigan. 480 pp.

Nieves H., G., J. A. Vázquez, H. Luquín S., E. Iracheta R. e Y. Vargas R. 1999. Plantas vasculares del norte de Jalisco y zonas adyacentes de Durango, Nayarit y Zacatecas. Mexicoa 1(1): 41-77.

Ortiz V., M. 1990. Datos climatológicos del estado de Zacatecas. Instituto Nacional de Investigaciones Forestales y Agropecuarias. Centro de Investigaciones Forestales y Agropecuarias de Zacatecas. Secretaría de Agricultura y Recursos Hidráulicos. Calera de Victor Rosales, Zacatecas. pp. 66-67.

Rohlf, F. J. 1998. NTSYS-pc, Numerical taxonomy and multivariate analysis system. Exeter Publishing, Ltd. Nueva York. 177 pp.

Riba, R. 1995. A manera de conclusión. In: Linares M., E., P. Dávila, F. Chiang C., R. Bye y T. Elias (eds.). Conservación de plantas en peligro de extinción. Diferentes enfoques. Instituto de Biología, Universidad Autónoma de México. México, D.F. pp. 171-175.

Rzedowski, J. 1957. Vegetación de las partes áridas de los estados de San Luis Potosí y Zacatecas. Rev. Soc. Mex. Hist. Nat. 8: 49-101.

Rzedowski, J. 1978. Vegetación de México. Editorial Limusa. México, D.F. 432 pp.

Rzedowski, J. 1993. Diversity and origins of the fanerogamic flora of Mexico. In: Ramamoorthy, T. P., R. Bye, A. Lot y J. Fa (eds.). Biological diversity of Mexico: Origins and distribution. Oxford University Press. Nueva York. pp. 129-144.

Rzedowski, J. y R. McVaugh. 1966. La vegetación de Nueva Galicia. Contr. Univ. Mich. Herb. 9: 1-123.

Sánchez, O. y G. López. 1988. A theoretical analysis of some indices of similarity as applied to biogeography. Folia Entomol. Mex. 75: 119-145.

Shannon, S. S. y W. V. Kramer. 1973. Geology of Sierra Santa Lucia and Sierra Papanton, Durango and Zacatecas, Mex. Soc. Geol. Mexicana. Bol. 34(1 y 2): 33-41. 
Acta Botanica Mexicana (2003), 64: 45-89

Weber, W. 1983. Species novae Bromeliacearum IV. Feddes Repert. 94: 609-611.

Recibido en agosto de 2001.

Aceptado en enero de 2003. 


\section{APÉNDICE 1}

Lista florística de la Sierra de Órganos, municipio de Sombrerete, Zacatecas, ordenada alfabéticamente por familia, género y especie para cada tipo de vegetación. La nomenclatura para familias dicotiledóneas y monocotiledóneas es de acuerdo con Cronquist (1988), para gimnospermas se sigue a McVaugh (1992) y para pteridofitas a Mickel (en McVaugh, 1992). Las abreviaturas en las columnas significan: $\mathrm{BQ}=$ Bosque de Quercus; $\mathrm{BP}=$ Bosque de Pinus; $\mathrm{BPQ}=$ Bosque de Pinus-Quercus; BPJ = Bosque de Pinus-Juniperus; VAP; Vegetación de arroyos pedregosos; VP = Vegetación de peñascos; $\mathrm{MMO}=$ Matorral de Mimosa-Opuntia; $\mathrm{P}$ = Pastizal; VA = Vegetación acuática y subacuática; V.I. = Valor de importancia acumulado. Los números dentro de las columnas indican las categorías de importancia asignadas para cada especie en cada tipo de vegetación: $4=$ Valor de importancia muy alto; 3 = Valor de importancia alto; 2 = Valor de importancia bajo; $1=$ Valor de importancia muy bajo. El 0 indica solamente presencia.

\begin{tabular}{llllll}
\hline Especies & BQ & BP & BPQ BPJ VAP VP MMO P VA V.I. \\
\hline
\end{tabular}

\section{PTERIDOPHYTA}

Adiantaceae

Cheilanthes bonariensis (Willd.) Proctor

Cheilanthes kaulfussii Kunze

Cheilanthes lendigera (Cav.) Sw.

Cheilanthes sinuata (Lag. ex Sw.) Domin

Pellaea cordifolia (Sessé \& Moc.) A. R. Sm.

Pellaea ternifolia (Cav.) Link

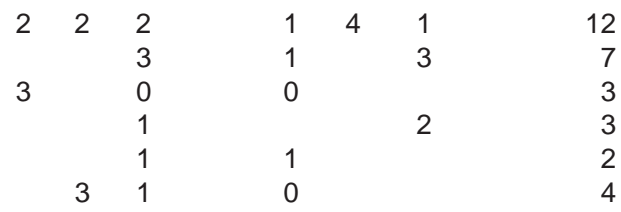

Aspleniaceae

Dryopteris rossii C. Chr.

0

Polypodiaceae

Polypodium thyssanolepis A. Br. ex Klotzsch

0

04

4

Selaginellaceae

Selaginella pallescens (Presl) Spring

Selaginella rupincola Underw.

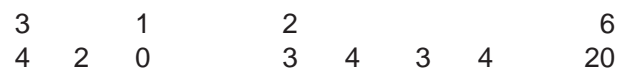

\section{GYMNOSPERMAE}

\section{Cupressaceae}

Juniperus deppeana Steud.

Juniperus flaccida Schltdl.

$\begin{array}{llll}3 & 2 & 4 & 4\end{array}$

02

12

7

3

4 
Acta Botanica Mexicana (2003), 64: 45-89

Apéndice. Continuación.

Especies

Pinaceae

Pinus cembroides Zucc.

Pinus chihuahuana Engelm.

\section{ANGIOSPERMAE}

\section{DICOTYLEDONEAE}

Acanthaceae

Dyschoriste decumbens (A. Gray) Kuntze Stenandrium dulce (Cav.) Nees

Amaranthaceae

Alternanthera caracasana Kunth

Amaranthus aff. hybridus L.

Gomphrena serrata L.

Guilleminea densa (Willd.) Moq.

Anacardiaceae

Rhus aromatica var. trilobata (Nutt.)

A. Gray ex S. Wats.

Rhus virens A. Gray (dos formas)

Apiaceae

Eryngium sp.

Hydrocotyle ranunculoides $\mathrm{L}$. $\mathrm{f}$.

Prionosciadium linearifolium (S. Wats.)

J. M. Coult. \& Rose

Apocynaceae

Telosiphonia hypoleuca (Benth.) Henr.

$0 \quad 0$

$\begin{array}{llll}4 & 3 & 3 & 3\end{array}$

0

3

4
BQ $B P$ BPQ BPJ VAP VP MMO P VA V.I.
23

3
2002
$\begin{array}{ll}0 & 2 \\ 0\end{array}$
6
0

$\begin{array}{lll}0 & 0 & 0 \\ 0 & 0 & 0\end{array}$

$\begin{array}{rrr}4 & 4 & 21 \\ & 4 & 5\end{array}$

Asclepiadaceae

Asclepias linaria Cav.

Matelea pedunculata (Decne.) Woods.

3

4

Sarcostemma cf. torreyi (A. Gray) Woods.

Asteraceae

Acourtia longifolia (S. F. Blake)

1

1

Reveal \& R. M. King

Adenophyllum porophylum (Cav.) Hemsl. 
Apéndice. Continuación.

Ageratina brevipes (DC.) R. M. King \& H. Rob.

Ageratina calaminthifolia (Kunth)

0

3

R. M. King \& H. Rob.

Ageratina espinosarum (A. Gray)

R. M. King \& H. Rob.

Ageratina espinosarum (A. Gray)

R. M. King \& H. Rob. var. subintegrifolia

(B. L. Rob.) B. L. Turner

Ageratina petiolaris (Moc. \& Sessé ex DC.)

R. M. King \& H. Rob.

Ageratina pichinchensis (Kunth)

R. M. King \& H. Rob.

Ageratina rubricaulis (Kunth)

R. M. King \& H. Rob.

Ageratum corymbosum Zuccagni ex Pers.

Artemisia ludoviciana Nutt.

Aster subulatus Michx.

Baccharis neglecta Britton

Baccharis occidentalis S. F. Blake

Baccharis pteronioides DC.

Baccharis salicifolia (Ruiz \& Pav.) Pers.

Bahia absinthifolia Benth.

Barkleyanthus salicifolius (Kunth)

H. Rob. \& Brettell

Berlandiera lyrata Benth.

Bidens angustissima Kunth

Bidens ferulifolia (Jacq.) DC.

Bidens odorata Cav.

Brickellia eupatorioides (L.) Shinners

Brickellia secundiflora (Lag.) A. Gray

Brickellia spinulosa (A. Gray) A. Gray

Brickellia vernicosa $B$. L. Rob.

Brickellia veronicifolia (Kunth) A. Gray

Carphochaete grahamii A. Gray

Cirsium mexicanum DC.

Conyza canadensis (L.) Cronquist

Coreopsis macvaughii D. J. Crawford

Coreopsis rudis (Benth.) Hemsl.

Cosmos parviflorus (Jacq.) Pers.

Chaetopappa bellioides (A. Gray) Shinners

Chaetopappa ericoides (Torr.) G. L. Nesom

Dahlia coccinea Cav.

Dahlia pinnata Cav.

Dahlia sp.

Dyssodia papposa (Vent.) Hitchc.

Dyssodia pinnata (Cav.) B. L. Rob.

Erigeron delphinifolius Willd.

Erigeron janivultus G. L. Nesom

Eutetras sp.

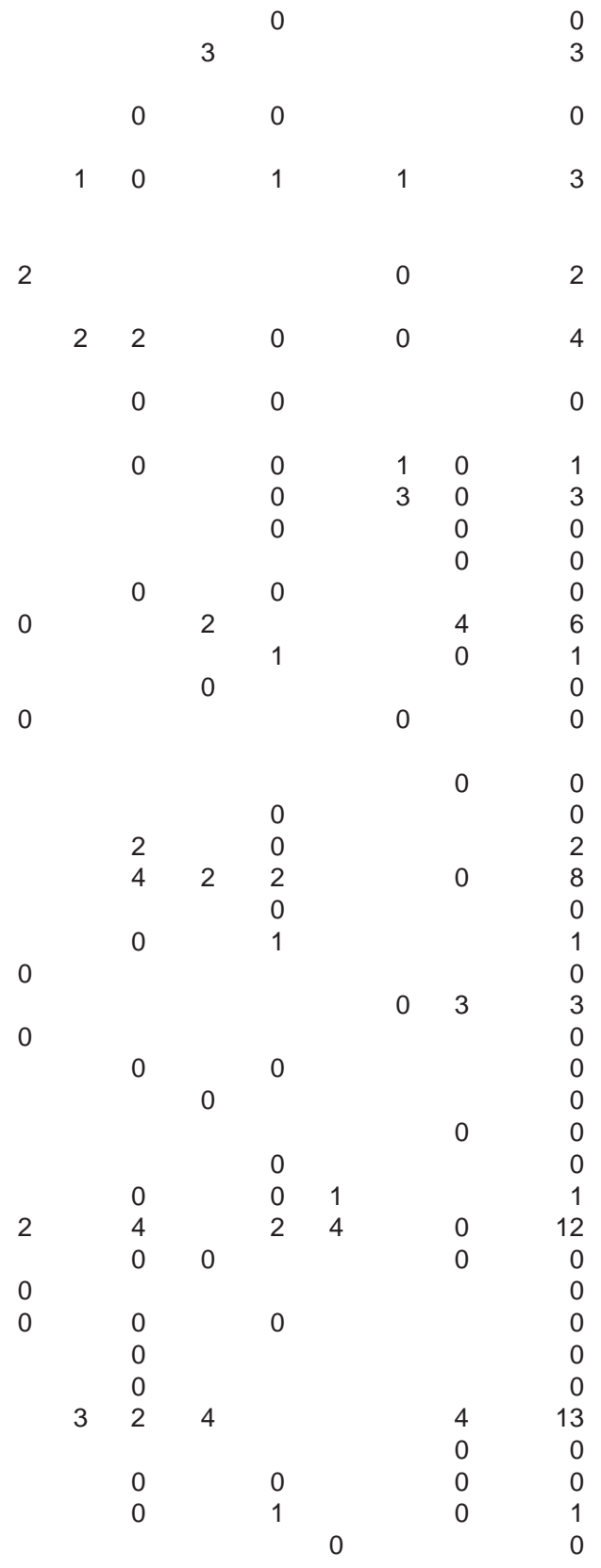


Acta Botanica Mexicana (2003), 64: 45-89

Apéndice. Continuación.

Especies

BQ $B P$ BPQ BPJ VAP VP MMO P VA V.I.

Gaillardia comosa A. Gray

Galinsoga parviflora Cav.

Grindelia oxylepis Greene

Gutierrezia conoidea (Hemsl.) M. A. Lane

Gutierrezia microcephala (DC.) A. Gray

Gutierrezia sericocarpa (A. Gray) M. A. Lane

Gymnosperma glutinosum (Spreng.) Less.

Heterosperma pinnatum Cav.

Heteroteca inuloides Cass.

Hieracium abscissum Less.

Hieracium pringlei A. Gray

Hymenoxys scaposa (DC.) Parker

Laennecia filaginoides DC.

Laennecia sophiifolia (Kunth) G. L. Nesom

Machaeranthera gymnocephala (DC.) Shinners

Melampodium sericeum Lag.

Milleria quinqueflora $\mathrm{L}$.

Montanoa leucantha (Lag. \& Segura)

S. F. Blake

Pectis prostrata Cav.

Perymenium mendezii DC.

Pinaropappus roseus (Less.) Less.

Psacalium amplum (Rydb.) H. Rob. \& Brettell

Psacalium peltatum (Kunth) Cass.

Psacalium sinuatum (Cerv.) H. Rob. \& Brettell

Pseudognaphalium attenuatum (DC.) Anderb.

Pseudognaphalium canescens (DC.) Anderb.

Pseudognaphalium inornatum (DC.) Anderb.

Psilactis brevilingulata Sch. Bip. ex Hemsl.

Roldana sessilifolia (Hook. \& Arn.)

H. Rob. \& Brettell

Sanvitalia angustifolia Engelm. ex A. Gray

Sanvitalia procumbens Lam.

Schkuhria pinnata (Lam.) Kuntze

Simsia amplexicaulis (Cav.) Pers.

Sinclairia palmeri (A. Gray) B. L. Turner

Stevia lucida Lag.

Stevia micrantha Lag.

Stevia ovata Willd.

Stevia porphyrea McVaugh

Stevia salicifolia Cav.

Stevia serrata Cav.

Stevia viscida Kunth

Steviopsis thyrsiflora (A. Gray) B. L. Turner

Tagetes lucida Cav.

Tagetes lunulata Ortega

Tagetes micrantha Cav.

Taraxacum officinale Wigg.

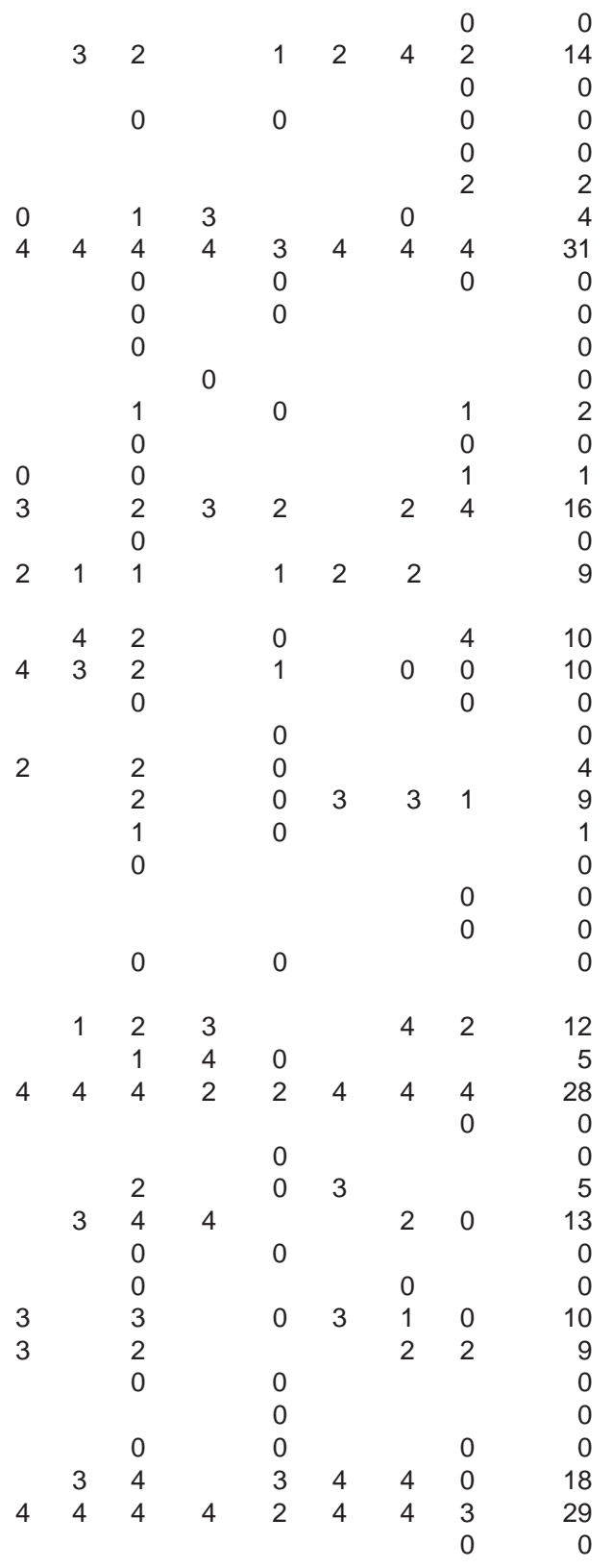


Apéndice. Continuación.

Tithonia tubiformis (Jacq.) Cass.

Tridax balbisioides (Kunth) A. Gray

Trixis angustifolia DC.

Thymophylla pentachaeta (DC.) Small

Verbesina pantoptera S. F. Blake

Verbesina serrata Cav.

Verbesina sp.

Viguiera cordifolia A. Gray

Viguiera dentata (Cav.) Spreng.

Viguiera hypargyrea Greenm.

Viguiera linearis (Cav.) Sch. Bip. ex Hemsl.

Xanthocephalum gymnospermoides

(A. Gray) Benth.

Zinnia bicolor (DC.) Hemsl.

Zinnia peruviana (L.) L.

Begoniaceae

Begonia gracilis Kunth

$\begin{array}{lll}4 & 4 & 4 \\ 3 & & \end{array}$

0

2

0

0

0

0

0

0

0

1

1

0

0

0

0

1

0

0 $\begin{array}{lll}0 & 0 & 0\end{array}$

$\begin{array}{lllll}0 & 4 & 4 & 3 & 23\end{array}$

$20 \quad 5$

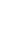

2

0

0

0

0

0

Boraginaceae

Lithospermum calycosum (Macbride) I. M. Johnst.

0

0

Brassicaceae

Brassica rapa L.

Lepidium virginicum $\mathrm{L}$.

Lesquerella mirandiana Rollins

Rorippa mexicana (Moc. \& Sessé) Standl.

Thelypodium wrightii (A. Gray) Rydb.

0

Buddlejaceae

Buddleja cordata Kunth

Buddleja scordioides Kunth

$\begin{array}{llllllll}0 & 2 & 0 & & 1 & 2 & & 3 \\ & & 0 & & & 0 & 2\end{array}$

Burseraceae

Bursera fagaroides (Kunth) Engl.

0

0

Cactaceae

Coryphantha sp.

Echinocereus polyacanthus Engelm.

Mammilaria gummifera Engelm.

Mammilaria moelleriana Boed.

Opuntia durangensis Britton \& Rose

$\begin{array}{rrrrrrrr} & & & & & & 0 & 0 \\ 2 & 1 & & & 3 & 0 & & 3 \\ & & & & 2 & 2 & 1 & 8 \\ 2 & 1 & 1 & 2 & 1 & 0 & & 2 \\ & & & & & & & \\ & & & & & & \end{array}$


Acta Botanica Mexicana (2003), 64: 45-89

Apéndice. Continuación.

Especies

$B Q \quad B P B P Q$ BPJ VAP VP MMO P VA V.I.

Opuntia imbricata (Haw.) DC.

Opuntia megacantha Salm-Dyck

Opuntia robusta $\mathrm{H}$. Wendl.

Stenocactus zacatecasensis (Britton \& Rose)

Berger ex Backeberg \& Knuth

Caesalpiniaceae

Senna crotalarioides (Kunth) Irwin \& Barneby

2

0

2

Campanulaceae

Lobelia fenestralis Cav.

Lobelia gruina Cav.

0

0

0

Lobelia laxiflora Kunth

0

2

0

0

2

Caryophyllaceae

Arenaria lanuginosa (Michx.) Rohrb.

Arenaria lycopodioides Willd. ex Schltdl.

Drymaria arenarioides Willd.

Drymaria leptophylla (Cham. \& Schltdl.) Fenzl

Drymaria tenuis S. Wats.

Drymaria xerophylla A. Gray

Silene laciniata Cav.

32

0

24

3

0

$\begin{array}{lll}4 & 3 & 4\end{array}$

Clusiaceae

Hypericum silenoides Juss.

Convolvulaceae

Dichondra argentea Humb. \& Bonpl. ex Willd.

Evolvulus alsinoides L.

Evolvulus prostratus Rob.

233

$\begin{array}{lll}4 & 3 & 2\end{array}$

Evolvulus sericeus Sw.

Ipomoea capillacea (Kunth) G. Don

Ipomoea durangensis House

Ipomoea madrensis S. Wats.

Ipomoea pubescens Lam.

Ipomoea purpurea (L.) Roth

Ipomoea stans Cav. $\begin{array}{llll}1 & 3 & 3 & 0\end{array}$

18

$0 \quad 0$

$\begin{array}{rrr}1 & 4 & 10 \\ 0 & 3 & 3 \\ & 0 & 0 \\ & & 6 \\ & 0 & 3 \\ & & \\ & & \end{array}$

0

0 
Apéndice. Continuación.

Crassulaceae

Echeveria mucronata (Bak.) Schltdl.

Sedum aff. glabrum (Rose) Praeger

Sedum sp.

0

1
0
0

Cucurbitaceae

Cucurbita sp.

Sicyos deppei G. Don

0

$0 \quad 0$

Cuscutaceae

Cuscuta glabrior (Engelm.) Yunck.

0

0

Chenopodiaceae

Atriplex muricata Humb. \& Bonpl. Chenopodium graveolens Willd.

Salsola tragus $\mathrm{L}$.

$\begin{array}{rrrrrrr} & 0 & & & & 0 & 0 \\ 1 & 3 & 2 & 1 & 3 & 13 \\ & & & & 0 & 0\end{array}$

\section{Ericaceae}

Arbutus arizonica (Sarg.) A. Gray Arctostaphylos pungens Kunth

$\begin{array}{rrrrrrr} & & 0 & 0 & & & 0 \\ & 1 & 3 & 3 & 2 & 1 & 13\end{array}$

\section{Euphorbiaceae}

Acalypha monostachya Cav.

Acalypha neomexicana Müll. Arg.

Acalypha phleoides Cav.

Croton dioicus Cav.

Euphorbia dentata Michx.

Euphorbia hirta L.

Euphorbia potosina Fernald

Euphorbia radians Benth.

Euphorbia wrightii Torr. \& A. Gray

Jatropha dioica Sessé ex Cerv.

Tragia nepetifolia Cav.

$\begin{array}{rrrrrrrrr} & & & 0 & & & & & 0 \\ 0 & & 3 & 4 & 1 & & 3 & & 14 \\ & & & & & & & & 0 \\ & & 1 & 2 & 0 & 3 & & & 0 \\ 4 & 4 & 4 & 4 & & 2 & 4 & 4 & 6 \\ & & & & & & 0 & 0 & 0 \\ & & & & & & & 0 & 0 \\ & & 0 & & 0 & & & & 0 \\ & & & & & & 4 & 0 & 4 \\ & & & & & & 2 & 2\end{array}$

Fabaceae

Amicia zygomeris DC.

Astragalus diphacus S. Wats.

Astragalus aff. wootonii Sheld.

Cologania angustifolia Kunth

Cologania obovata Schltdl.

Dalea bicolor Humb. \& Bonpl. ex Willd.

$\begin{array}{rrrrrr} & 0 & & & & 0 \\ & & 0 & & & 0 \\ 3 & & 0 & & & 0 \\ & 3 & 4 & 1 & & 11 \\ 0 & 0 & & & & 0 \\ & 0 & 0 & & 2 & 2\end{array}$


Acta Botanica Mexicana (2003), 64: 45-89

Apéndice. Continuación.

Dalea humilis G. Don

Dalea leucostachys A. Gray

Dalea prostrata Ortega

Desmodium neomexicanum A. Gray

Desmodium retinens Schltdl.

Erythrina montana Rose \& Standl.

Eysenhardtia polystachya (Ortega) Sarg.

Indigofera montana Rose

Lotus oroboides (Kunth)

Ottley ex Kearney \& Peebles

Lupinus mexicanus Cerv. ex Lag.

Macroptilium gibbosifolium (Ortega) A. Delgado

Nissolia wislizeni (A. Gray) A. Gray

Phaseolus acutifolius A. Gray

Phaseolus ritensis M. E. Jones

Trifolium goniocarpum Lojac.

Zornia thymifolia Kunth

Fagaceae

Quercus depressipes Trel.

Quercus durifolia Seem.

Quercus eduardi Trel.

Quercus grisea Liebm.

Quercus laeta Liebm.

Quercus aff. microphylla Née

Quercus potosina Trel.

Quercus aff. rugosa Née

Quercus sp.

\section{Garryaceae}

Garrya wrightii Torr.

\section{Geraniaceae}

Erodium cicutarium (L.) L'Hér. ex Aiton

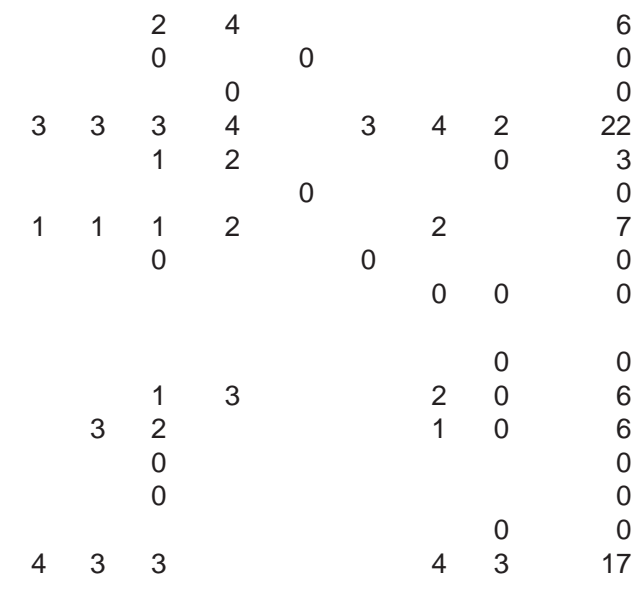

\section{Lamiaceae}

Origanum sp.

Salvia axillaris Moc. \& Sessé ex Benth.

Salvia greggii Gray

Salvia aff. laevis Benth.

Salvia lycioides A. Gray

Salvia nana Kunth

Salvia prunelloides Kunth 
Apéndice. Continuación.

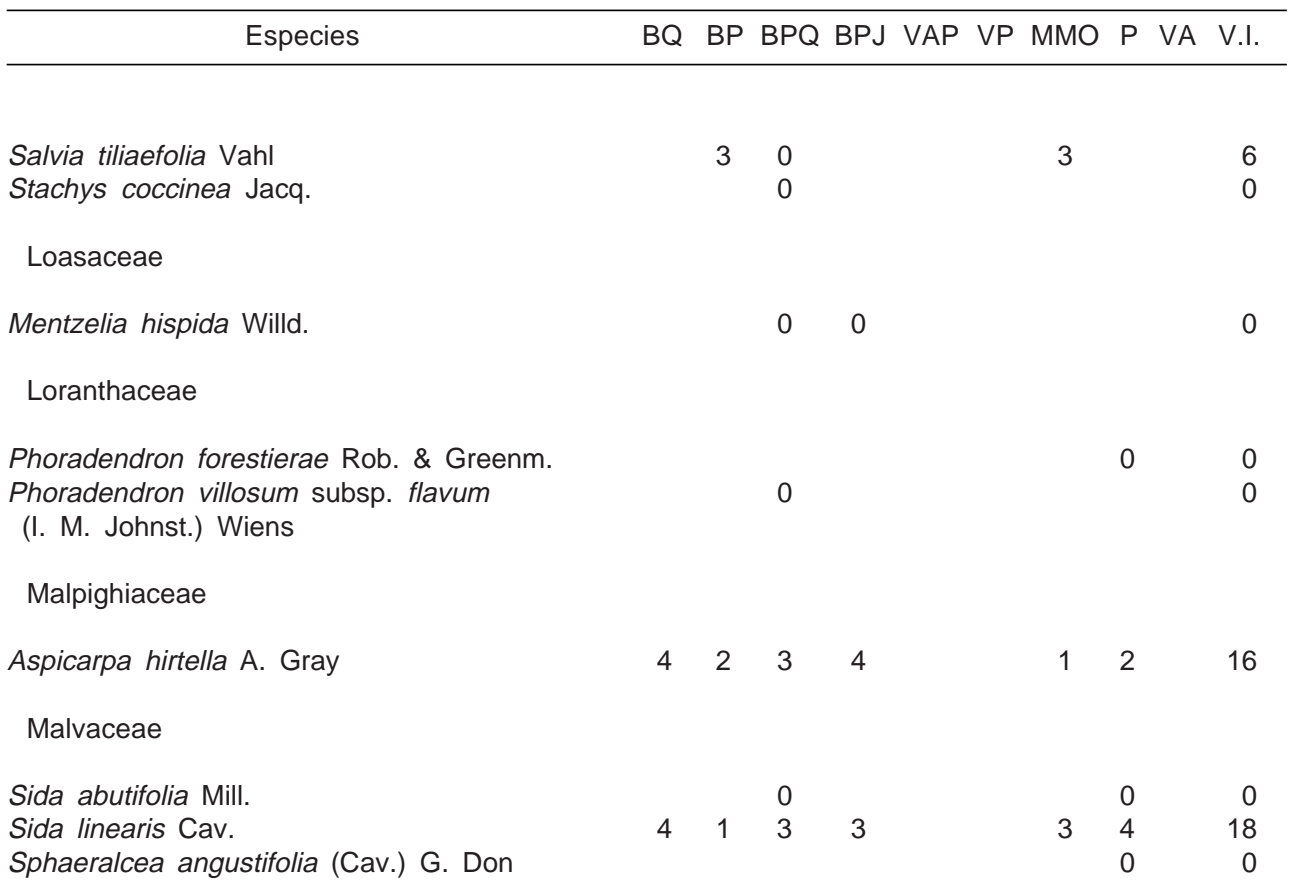

Mimosaceae

Acacia angustissima (Mill.) Kuntze

Acacia schaffneri (S. Watson) F. J. Herm.

Calliandra eriophylla Benth.

Calliandra humilis (Schltdl.) Benth.

Mimosa aculeaticarpa Ortega

Mimosa dysocarpa Benth.

Prosopis laevigata (Willd.) M. C. Johnst.

0

$\begin{array}{rrrrrrrrr}0 & & & & 0 & & & & 0 \\ & 2 & & 3 & & & & 3 & 8 \\ & 4 & 3 & & & & & & 7 \\ & & 2 & & & & & 3 & 5 \\ 4 & 3 & 4 & & 1 & 1 & 4 & 4 & 21 \\ 4 & & 1 & 3 & & & 2 & 1 & 11 \\ & & & & & & & 0 & 0\end{array}$

Nyctaginaceae

Oxybaphus comatus (Small) Weatherby

Oxybaphus glabrifolius (Ortega) Vahl

Oxybaphus linearis (Pursh) Heimerl

Oleaceae

Forestiera durangensis Standl.

0

3

0

2

5

Fraxinus velutina Torr.

Onagraceae

Gaura drummondii (Spach) Torr. \& A. Gray Lopezia racemosa Cav.

0

$0 \quad 0$

0 
Acta Botanica Mexicana (2003), 64: 45-89

Apéndice. Continuación.

Especies

Oenothera pubescens Willd. ex Spreng.

Oenothera rosea L'Hér. ex Aiton

Oxalidaceae

Oxalis alpina (Rose) Knuth

Oxalis corniculata L.

Oxalis decaphylla Kunth

Oxalis sp.

Phytolaccaceae

Phytolacca icosandra L.

Piperaceae

Peperomia campylotropa Hill

2

$\begin{array}{lll}1 & 3 & \\ 1 & & 3\end{array}$

0

0

$0 \quad 0$

0

Plantaginaceae

Plantago linearis Kunth

33

$\begin{array}{lll}4 & 3 & 3\end{array}$

16

Plumbaginaceae

Plumbago pulchella Boiss.

0

0

Polemoniaceae

Ipomopsis pinnata (Cav.) V. Grant

Loeselia coerulea (Cav.) G. Don

Loeselia mexicana (Lam.) Brand

Loeselia scariosa (Mart. \& Gal.) Walp.

Polygalaceae

Polygala alba Nutt.

Polygala rivinifolia Kunth

Polygala sp.

$\begin{array}{lllll}2 & 2 & 3 & 2 & 1 \\ & & & & 0\end{array}$

$\begin{array}{rrr} & 0 & 0 \\ 2 & 3 & 15 \\ & & 0\end{array}$

Polygonaceae

Polygonum hydropiperoides Michx.

$0 \quad 0$

Portulacaceae

Portulaca oleracea L.

Portulaca pilosa L.

Talinum humile Greene

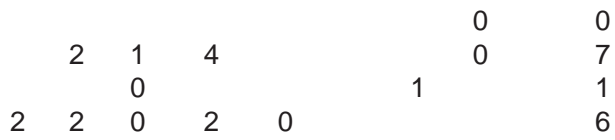

7
1
6


Apéndice. Continuación.

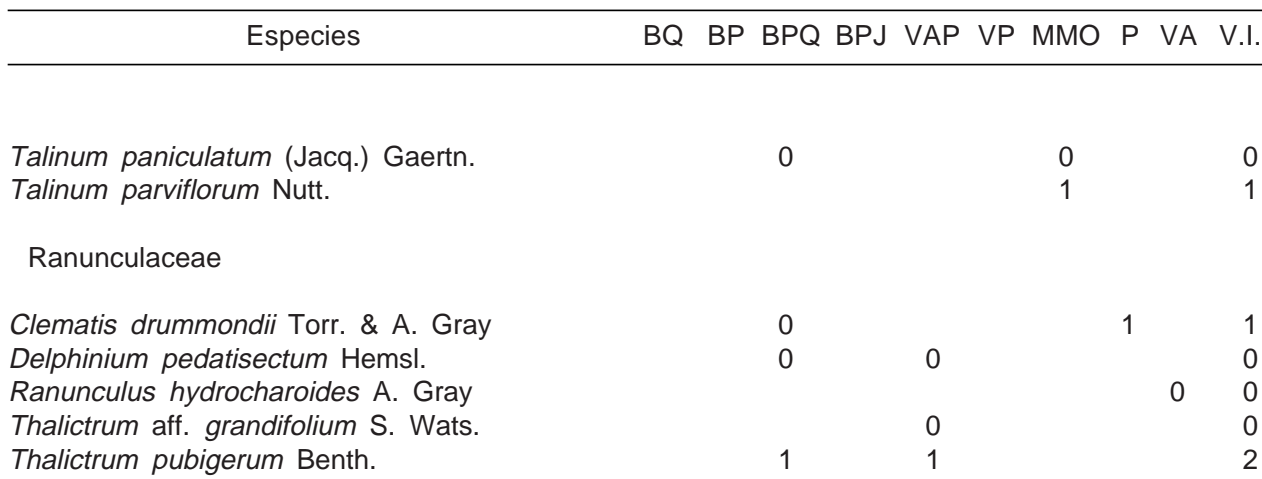

Resedaceae

Reseda luteola L.

Rhamnaceae

Ceanothus greggii A. Gray

var. lanuginosus Jones

Condalia ericoides (A. Gray) M. C. Johnst.

Rosaceae

Amelanchier denticulata (Kunth) Koch

Cowania mexicana D. Don

0

Holodiscus dumosus (Nutt.) Heller

Potentilla sp.

Prunus serotina Ehrh.

Rubiaceae

Bouvardia scabrida Mart. \& Gal.

Bouvardia ternifolia (Cav.) Schlecht.

Crusea diversifolia (Kunth) W. R. Anderson

Crusea longiflora (Willd. ex Roem. \& Schult.)

0

$\begin{array}{rrrrrrrr} & 0 & & 1 & 2 & 2 & 0 & 5 \\ 2 & 4 & 4 & 1 & 4 & 1 & 4 & 0 \\ 3 & 2 & & 0 & & 1 & & 6\end{array}$

W. R. Anderson

Galium mexicanum Kunth

0

Houstonia rubra A. Gray

Houstonia wrightii A. Gray

0

Richardia tricocca (Torr. \& A. Gray) Standl.

Spermacoce verticillata $\mathrm{L}$.

13 0

$$
0
$$$$
6
$$

Salicaceae

Salix nigra Marshall

Scrophulariaceae

Bacopa procumbens (Mill.) Greenm. 
Acta Botanica Mexicana (2003), 64: 45-89

Apéndice. Continuación.

Especies

Castilleja tenuiflora Benth.

Lamourouxia rhinanthifolia Kunth

Mimulus glabratus Kunth

Penstemon campanulatus (Cav.) Willd.

Solanaceae

Datura stramonium L.

Nicandra physalodes (L.) Gaertn.

Physalis chenopodiifolia Lam.

Physalis philadelphica Lam.

Solanum nigrum L.

Solanum rostratum Dunal

Verbenaceae

Citharexylum aff. rosei Greenm.

Lippia durangensis Mold.

Priva mexicana (L.) Pers.

Verbena menthaefolia Benth.

Violaceae

Viola barroeteana Schaffn.

MONOCOTYLEDONEAE

\section{Agavaceae}

Agave parryi Engelm.

Agave schidigera Lem.

Prochnyanthes mexicana (Zucc.) Rose

Yucca decipiens Trel.

Bromeliaceae

Tillandsia fresnilloensis W. Weber \& Ehlers

Tillandsia recurvata (L.) L.

Commelinaceae

Commelina dianthifolia Delile

Cymbispatha commelinoides (Roem. \& Schult.)

Pichon

Gibasis linearis (Benth.) Rohweder

Tradescantia crassifolia Cav.

Tripogandra purpurascens (Schauer) Handlos
BQ $B P$ BPQ BPJ VAP VP MMO P VA V.I.

$0 \quad 0$

0

0

0

0

3

$$
2
$$

$\begin{array}{lll}1 & 0 & 2 \\ 0 & \end{array}$

$0 \quad 0$

0

0 
Apéndice. Continuación.

\section{Cyperaceae}

Bulbostylis arcuata Kral Bulbostylis juncoides (Vahl) Kük.

Carex planostachys Kunze

Cyperus aggregatus (Willd.) Endl.

Cyperus aff. mutisii (Kunth) Griseb.

Cyperus niger Ruiz et Pav.

Cyperus pallidicolor (Kük.) G. C. Tucker

Cyperus seslerioides Kunth

Cyperus spectabilis Link

Cyperus sphaerolepis Boeck.

Cyperus squarrosus L.

Eleocharis cf. macrostachya Britton

Eleocharis montevidensis Kunth

Eleocharis sp.

Karinia mexicana (Britton) Reznicek \& McVaugh

$\begin{array}{lll}0 & 0 \\ 0 & 0\end{array}$

$0 \quad 0$

$0 \quad 0$

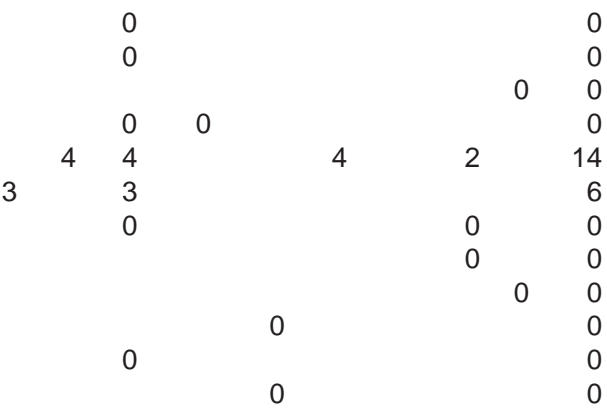

Iridaceae

Nemastylis tenuis (Herb.) S. Watson

Sisyrinchium convolutum Nocca

Sisyrinchium tenuifolium Humb. \& Bonpl. ex Willd.

Juncaceae

Juncus acuminatus Michx.

Juncus arcticus Willd.

0

0

1

$0 \quad 1$

Lemnaceae

Lemna gibba L.

$0 \quad 0$

Liliaceae

Allium glandulosum Link \& Otto

Asphodelus fistulosus $\mathrm{L}$.

320

10

22

Milla biflora Cav.

Nothoscordum bivalve (L.) Britton

Melanthiaceae

Schoenocaulon texanum Scheele

$\begin{array}{lllll} & 3 & & 0 & 8 \\ 0 & & 0 & 0 & 0 \\ & & & & 1 \\ 1 & & 0 & & 5 \\ & 2 & 1 & 0 & 4 \\ & & & 0 & 0\end{array}$

0 
Acta Botanica Mexicana (2003), 64: 45-89

Apéndice. Continuación.

Especies

$B Q \quad B P B P Q$ BPJ VAP VP MMO P VA V.I.

Nolinaceae

Dasylirion wheeleri S. Wats. ex Rothr.

Nolina juncea (Zucc.) J. Macbr.

$\begin{array}{lllll}3 & 1 & 0 & 0 & 4\end{array}$

0

Poaceae

Aegopogon tenellus (DC.) Trin.

Agrostis hyemalis (Walt.) Britton, Stearn \& Pogg.

Aristida adscensionis L.

Bothriochloa barbinodis (Lag.) Herter

Bouteloua aristidoides (Kunth) Griseb.

Bouteloua curtipendula (Michx.) G. S. Torr.

Bouteloua gracilis (Kunth) Lag.

$\begin{array}{lll}0 & 0 & 0\end{array}$

Bouteloua hirsuta Lag.

Brachiaria meziana Hitchc.

Brachypodium mexicanum (Roem. \& Schult.) Link

$\begin{array}{rrrrrrrrr} & & 2 & & 0 & & & & \\ 3 & 3 & 4 & 4 & 1 & 4 & 4 & 0 & 23 \\ & & 0 & 4 & 0 & & & & 4 \\ 0 & & & & & & 3 & & 3 \\ 2 & 3 & & 4 & & & & 3 & 12 \\ 4 & 3 & 4 & 0 & & & 4 & 4 & 19 \\ & & & 0 & & & & 0 & 0\end{array}$

Bromus carinatus Hook. \& Arn.

Cathestecum brevifolium Swallen

Cynodon dactylon (L.) Pers.

Chloris virgata Sw.

Eleusine multiflora Hochst. ex A. Rich.

Eragrostis intermedia Hitchc.

Eragrostis mexicana (Hornem.) Link

Eragrostis pectinacea (Michx.) Nees

Hilaria cenchroides Kunth

Leptochloa dubia (Kunth) Nees

Lycurus phleoides Kunth

Microchloa kunthii Desv.

Muhlenbergia alamosae Vasey

Muhlenbergia emersleyi Vasey

Muhlenbergia dubia E. Fourn.

Muhlenbergia montana (Nutt.) Hitchc.

Muhlenbergia polycaulis Scribn.

Muhlenbergia pubescens (Kunth) Hitchc.

Muhlenbergia rigida (Kunth) Trin.

0

2

Muhlenbergia speciosa Vasey

Muhlenbergia tenuifolia (Kunth) Kunth

Panicum bulbosum Kunth

Paspalum sp.

Piptochaetium fimbriatum (Kunth) Hitchc.

Polypogon elongatus Kunth

Rhynchelytrum repens (Willd.) C. E. Hubb.

Schizachyrium sp.

Setaria geniculata (Lam.) Beauv.

0

$0 \quad 0$

Setaria grisebachii Fourn.

Sporobolus trichodes Hitchc.

Stipa eminens Cav.

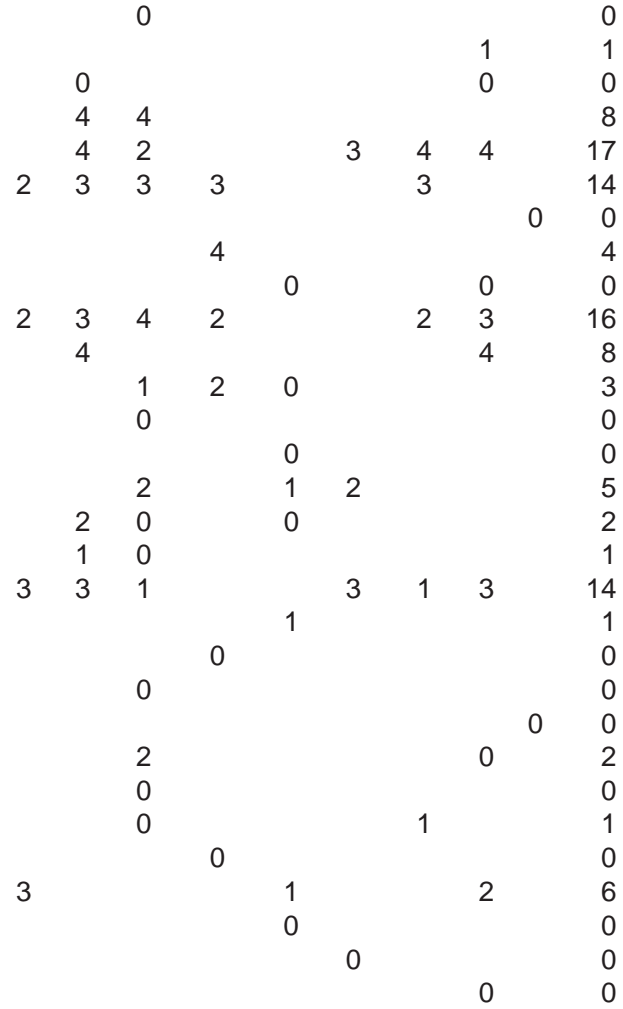


Enríquez et al.: Flora y vegetación de la Sierra de Órganos, Zacatecas

Apéndice. Continuación.

Especies BQ $\quad B P \quad B P Q$ BPJ VAP VP MMO P VA V.I.

Trachypogon plumosus

0

0

(Humb. \& Bonpl. ex Willd.) Nees

Tragus berteronianus Schult.

$0 \quad 0$

Pontederiaceae

Heteranthera peduncularis Benth. 


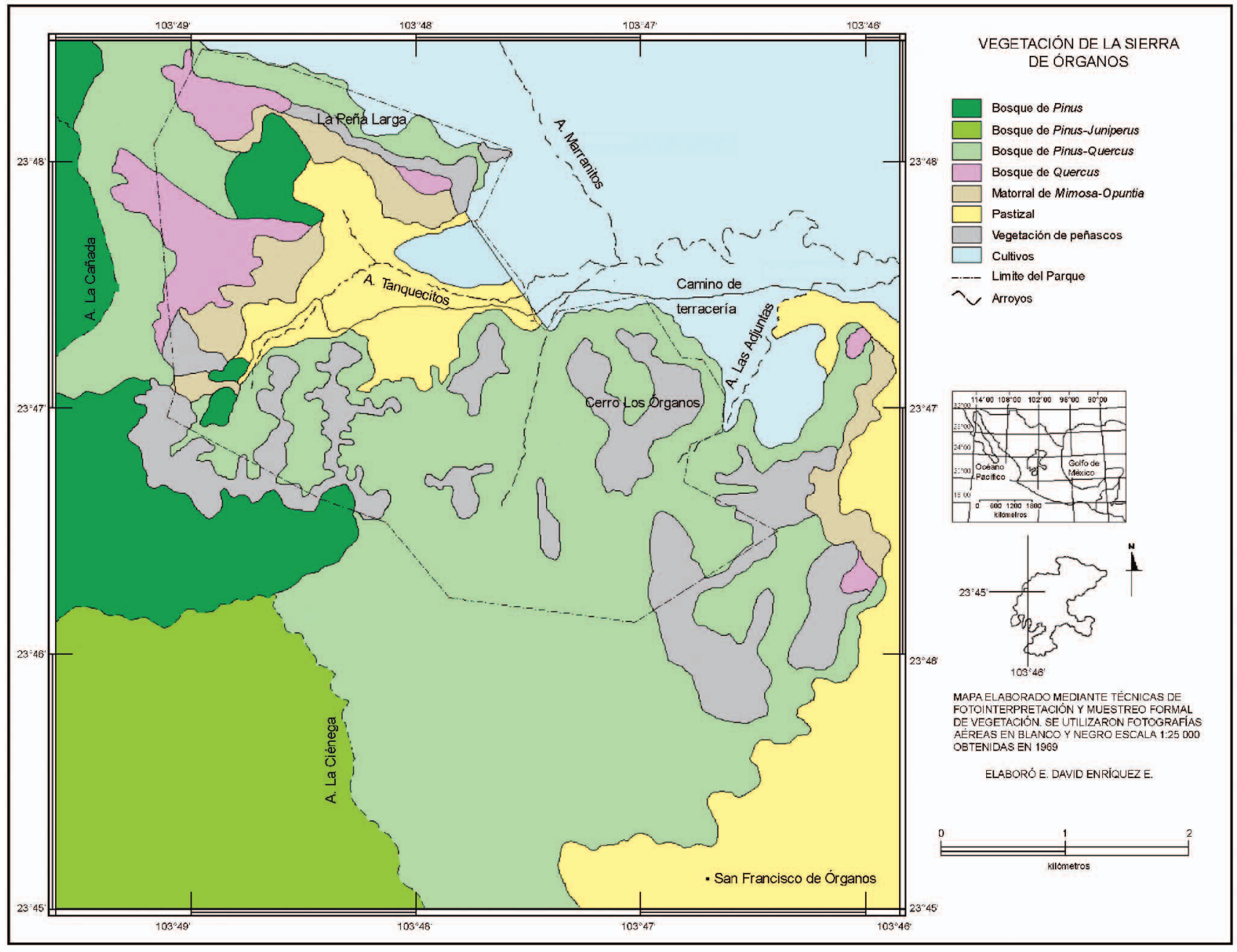

